\title{
analyses du comportement des milieux granulaires basées sur leur nature discontinue
}

\author{
par \\ B. Cambou \\ Maître assistant. École centrale de Lyon
}

\section{Introduction}

"The way out of the difficulty lies in dropping the old fundamental principles and starting again from the elementary fact that the sand consists of individual grains*."

K. Terzaghi, le premier (1920) a bien vu la nécessité d'une analyse au niveau de la particule. Cependant la complexité d'une telle étude a retardé ce type d'analyse alors que l'approche classique par la mécanique des milieux continus s'est, elle, considérablement développée. Cette dernière reste jusqu'à présent suffisante pour décrire convenablement le comportement global des milieux granulaires.

Cependant aujourd'hui l'utilisation de gros ordinateurs, d'une part, autorise la prise en compte de lois de comportement de plus en plus affinées, d'autre part nous donne l'outil indispensable à l'étude du milieu discontinu.

Ce type d'étude semble donc trouver aujourd'hui sa justification et l'outil qui lui faisaient défaut jusqu'à présent.

Nous présentons ici une revue sommaire des différents types d'approches que nous avons pu rencontrer dans la littérature ainsi que les résultats d'une étude personnelle.

\section{Analyse bibliographique}

Devant la complexité du problème les auteurs ont, dans la majorité des cas, cherché une approche simplifiée, nous avons pu distinguer 5 approches différentes :

(*) «Pour résoudre la difficulté il faut laisser tomber les vieux principes et repartir de la réalité élémentaire à savoir que le sable est constitué de grains individuels n, K. Terzaghi, 1920.

\subsection{Analyse expérimentale}

Cette analyse comporte deux aspects :

- Étude géométrique des assemblages d'un milieu granulaire

Cette étude a été surtout menée sur des matériaux bidimensionnels (petits rouleaux), nous citerons entre autres les travaux de MM. Biarez (1962) et Wiendieck (1964), Matsuoka (1974), Oda et Konishi (1974).

D'autres expérimentateurs ont travaillé sur des matériaux analogiques à 3 dimensions (billes) ou des matériaux réels (graviers), (Smith, Foot, Busang, 1929), (Field, 1963), (Marsal, 1973).

Ces diverses études montrent l'existence d'une anisotropie du milieu granulaire qui évolue en cours de sollicitation (fig. 1).

Elles ont aussi permis de proposer diverses formules liant le nombre moyen de contacts par particule et l'indice des vides. Field a proposé par exemple

$$
N=\frac{12}{1+e} \text {. }
$$

Fig. 1 Mesure de l'anisotropie à partir des mesures d'orientation des plans tangents de contact (BiarezWiendieck 1964)

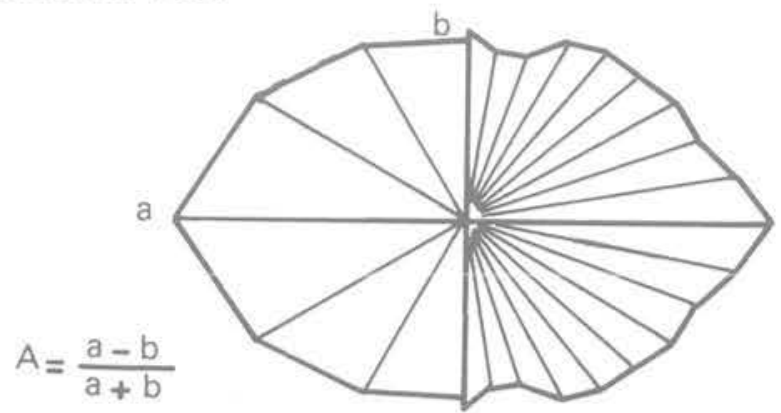


- Étude de la distribution des forces de contact

Cette étude a essentiellement été développée à partir de matériaux bidimensionnels photoélastiques. Nous citerons en particulier les travaux de MM. Dantu (1957, 1961), Weber (1966), De Josselin de Jong et Verruijt (1969), Schlosser (1974). Ces auteurs ont montré que la distribution des forces de contact n'est pas uniforme dans la structure; ils ont mis en évidence l'existence de "chainons de contraintes" composés de particules très sollicitées qui s'orientent en général suivant la direction principale majeure (fig. 2). Ils ont aussi montré que le nombre de contacts par particule augmente avec la contrainte moyenne appliquée.

On notera que cette technique a été utilisée pour l'étude de matériaux à 3 dimensions par Drescher (1966) qui confirme les résultats précédents.

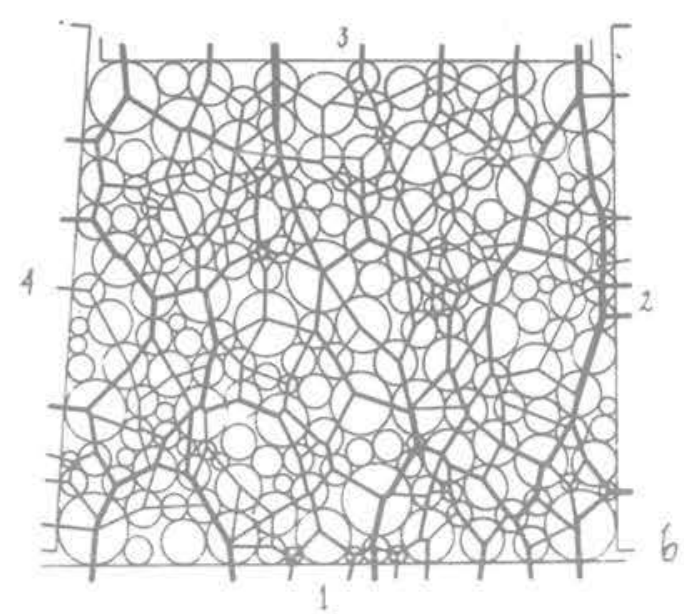

Fig. 2 Forces de contact dans une structure granulaire sollicitée (de Josselin, de Jong et Verruijt 1969)

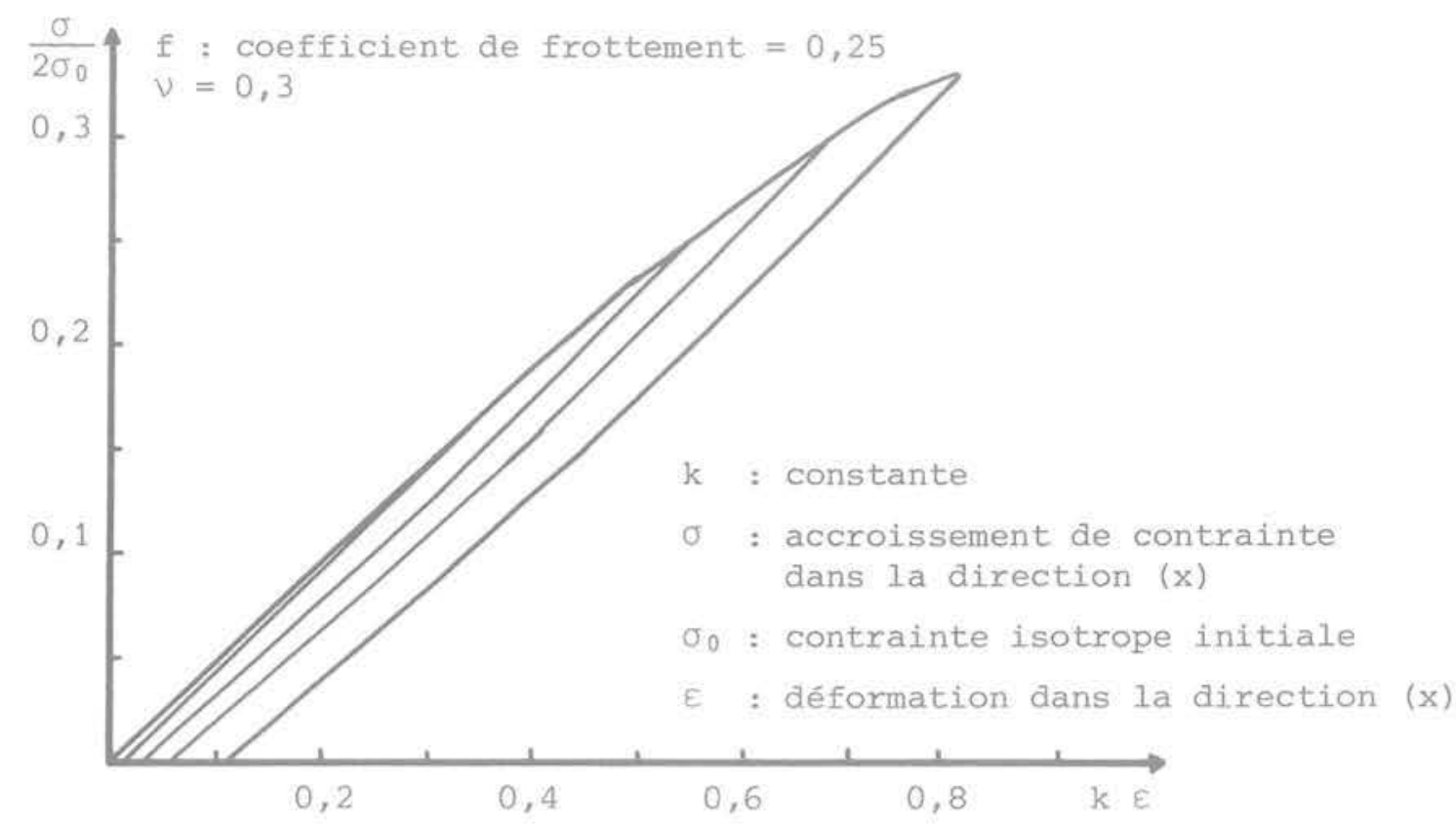

Fig. 3 Cycle de sollicitation dans un assemblage cubique simple sollicité suivant une direction $(x)$ parallèle à l'un de ses plans diagonaux (Deresiewicz 1958)

\subsection{Analyse théorique de l'équilibre statique d'une structure granulaire sans glissement}

Certains auteurs se sont intéressés à l'étude des forces de contact dans un assemblage régulier de sphères ou de cylindres. Ces différentes études sont basées sur les résultats obtenus par Hertz, Mindlin et Johnson concernant la déformation au contact entre deux sphères ou cylindres. Nous citerons les travaux de Deresiewicz (1958), Duffy et Mindlin (1957), Biarez (1961), Schlosser (1974),

Ces auteurs montrent que les relations contraintesdéformations sont non linéaires; en particulier lors d'une compression isotrope ils démontrent la relation $\varepsilon=k \sigma^{2 / 3}$ qui est en bon accord avec les résultats expérimentaux.

Pour des cycles de sollicitation ces études mettent en évidence l'existence d'une hystérésis ainsi que d'une certaine irréversibilité. Ces deux phénomènes sont liés aux déformations dues aux composantes tangentielles des forces de contact, en effet celles-ci entrainent des glissements dans la zone de contact, d'où une perte d'énergie (hystérésis) et une irréversibilité (fig. 3).
Ces divers résultats permettent d'analyser de façon très correcte le comportement expérimental de milieux réels pour des sollicitations où les glissements entre particules sont faibles (compressibilité isotrope, $n^{\text {ième }}$ cycle de sollicitation).

2.3 Analyse théorique des glissements dans une structure granulaire formée de particules rigides

Dans ce type d'analyse les auteurs considèrent que les déformations aux contacts sont, en première approximation négligeables et ne prennent en compte que les déformations par glissement entre particules. Ils considèrent généralement que les glissements se produisent en tous les points de contact.

Nous citerons essentiellement les travaux de Newland et Allely (1957), de Rowe (1962), de Bishop (1966), de Wilkins (1970).

Ces divers travaux ont permis d'analyser de façon intéressante l'écoulement plastique, de plus ils ont mis en évidence l'existence du phénomène de dilatance des matériaux granulaires (variation de volume sous une sollicitation déviatorique). 
Ainsi, Rowe, pour un contact glissant a pu écrire (fig. 4) :

$$
\frac{F_{1}}{F_{3}}=\operatorname{tg}(\Phi \mu+\beta)
$$

$\Phi_{\mu}$ : angle de frottement du matériau des particules;

au cours du glissement le rapport de l'énergie développée par $F_{1}$ et $F_{3}$ sera :

$$
E=\frac{\operatorname{tg}\left(\Phi_{\mu}+\beta\right)}{\operatorname{tg} \beta} .
$$

Rowe fait l'hypothèse que les contacts glissants sont tels que :

$$
\frac{\partial E}{\partial \beta}=0 .
$$

Il aboutit ainsi à la relation contrainte-dilatance :

$$
\frac{\sigma_{i}^{\prime}}{\sigma_{3}^{\prime}}=\left(1+\frac{\partial \varepsilon_{v}}{\partial \varepsilon_{1}}\right) \operatorname{tg}\left(\frac{\pi}{4}+\frac{\Phi \mu}{2}\right)
$$

$\varepsilon_{v}$ : déformation volumique $=\varepsilon_{1}+\varepsilon_{2}+\varepsilon_{3}$.

Pour obtenir une meilleure concordance avec les résultats expérimentaux, Rowe substitua l'angle $\Phi_{t}$ à $\Phi \mu$ avec $\Phi_{\mu}<\Phi_{1}<\Phi_{c v}$ (angle de frottement interne à volume constant).

Ce type d'analyse rend compte de façon satisfaisante du comportement des matériaux réels soumis à des contraintes déviatoires.

\subsection{Analyse théorique du comportement basée sur une approche statistique}

La plupart de ces études analysent essentiellement le glissement entre particules, elles prennent pour hypothèse que les contacts ne sont pas tous glissants simultanément. Horne (1969) à partir du concept de "Chemin solide moyen projeté", en utilisant les distributions des orientations de contact, a permis l'analyse de milieux hétérogènes et anisotropes. Murayama et Masuoka (1973) à partir d'hypothèses sur les évolutions des distributions des orientations de contacts analysent de façon intéressante un essai de cisaillement. Oda (1974) et Marsal (1973) font de plus des hypothèses sur les distributions probables des forces de contact, ils aboutissent à une analyse très poussée du comportement des milieux granulaires, ce type d'approche atteint un degré de complexité élevé. Wilson (1962) et Fletcher (1971) ont fait des hypothèses sur la distribution probable des déplacements des particules.

Nous signalons l'étude de Litwiniszyn (1974) qui considère les déplacements des particules ou des vides comme des processus stochastiques, ce type d'analyse s'applique par exemple à la détermination de la déformation en surface due à l'ouverture d'une cavité en profondeur.

Enfin signalons les travaux de certains auteurs (Mogami, 1969, Jowitt et Munro, 1975) qui ont tenté de faire une analyse thermodynamique des milieux granulaires basée sur l'hypothèse d'une entropie liée à une définition statistique de la géométrie du milieu, ce type d'analyse est très controversée.

\subsection{Analyses générales numériques}

L'utilisation de gros moyens informatiques permet actuellement une analyse plus complète du comportement des milieux granulaires.

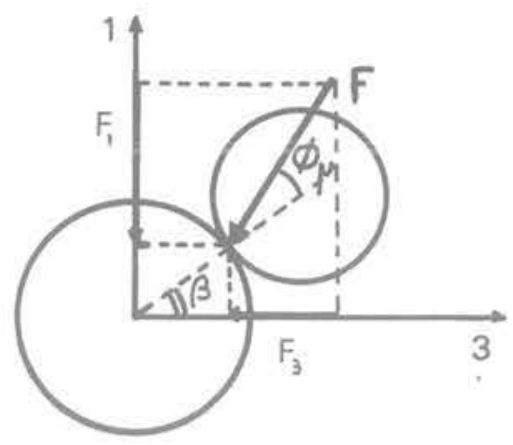

Fig. 4 Contact glissant dans un milieu granulaire

A part la méthode présentée par l'auteur (Cambou, 1979) nous citerons deux analyses très différentes:

\subsubsection{Une analyse complète de l'équilibre global d'un assemblage quelconque de cylindres ou de sphères prenant en compte les glissements entre particules présentée par Rodriguez Ortiz (1974)}

Ce type d'analyse rigoureuse et complète est très intéressant, son inconvénient réside dans le nombre limité de particules qui peuvent être analysées par le programme (fig. 5).

\subsubsection{Une analyse dynamique du système avant son état d'équilibre statique présentée par Cundall (1979)}

L'auteur discrétise le temps en petits intervalles $\Delta t$, les forces appliquées $F_{1}$ pendant ces intervalles de temps communiquent une certaine accélération $\gamma_{\mathrm{i}}$ aux particules $\mathrm{i}$, qui se traduit par un déplacement $\delta_{i}$. Les déplacements entraînent à leur tour l'apparition de forces aux contacts entre les particules $i$ et $j$ définies par une relation du type $F_{1 j}=K\left(\delta_{i}-\delta_{j}\right)$. Le processus est ainsi poursuivi jusqu'à l'obtention de l'équilibre global.

$\mathrm{Ce}$ genre d'analyse semble particulièrement bien adapté pour décrire certains phénomènes progressifs (rupture d'une pente en enrochement, explosion dans une cavité, etc.) (fig. 6).

\section{Analyse probabiliste du comportement d'un milieu granulaire à partir d'un modèle numérique}

Nous avons basé notre analyse du comportement des milieux granulaires sur un modèle numérique prenant en compte un certain nombre d'hypothèses probabilistes. Notre travail a essentiellement comporté deux étapes.

\subsection{Modèle numérique de mise en place d'un milieu granulaire}

L'utilisation de l'ordinateur permet de construire et d'analyser de façon très précise une structure granulaire. Ce type d'approche numérique a été utilisé par Auvinet $(1975,1977)$ et Rodriguez Ortiz (1974); le programme que nous avons développé, indépendamment de ces 2 auteurs, permet de construire des structures granulaires à deux et trois dimensions (formées de cylindres ou de sphères). 


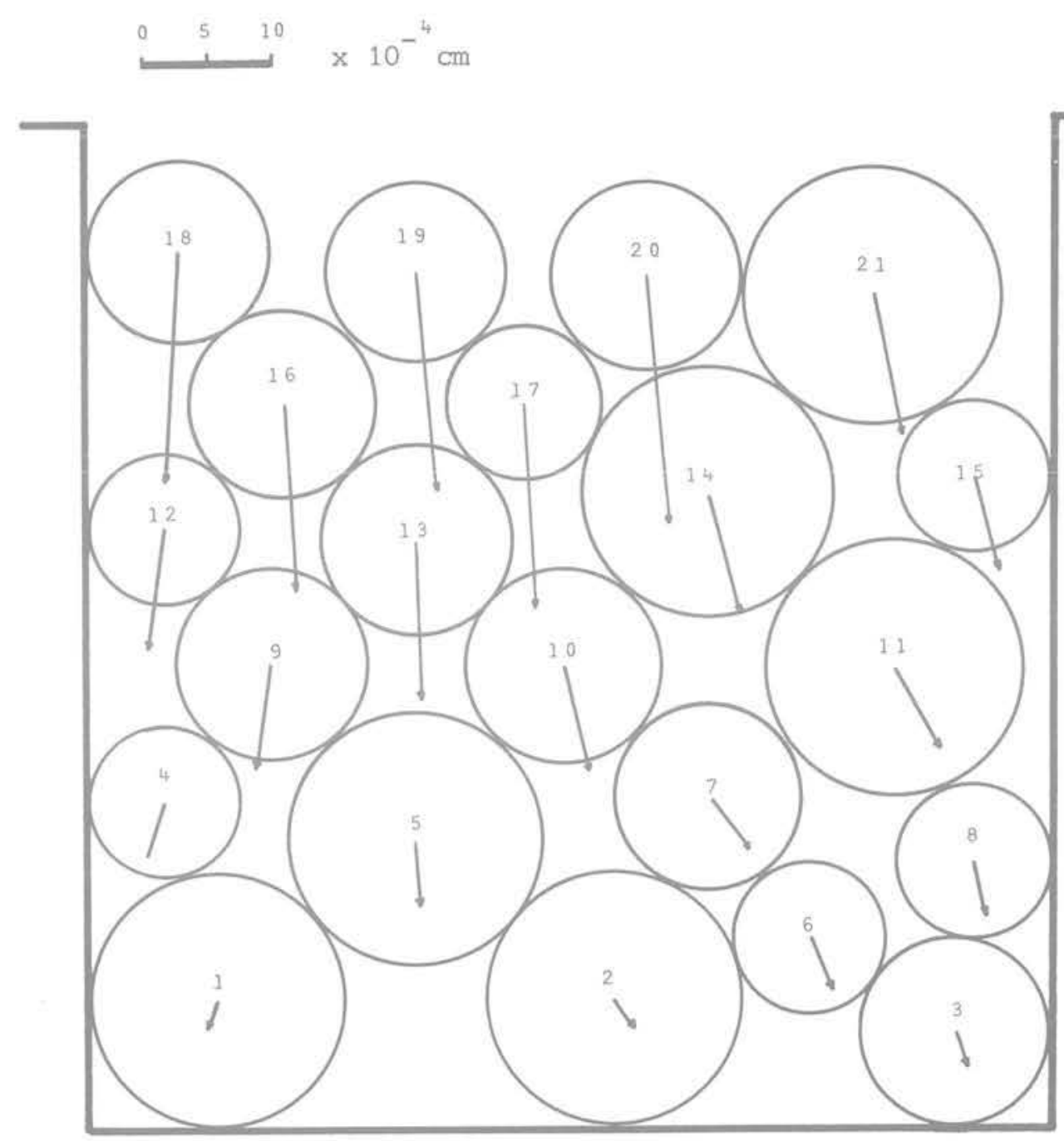

Fig. 5 Déplacements des particules dus à l'application d'une force verticale de $100 \mathrm{~N}$ sur chacune des particules supérieures (Rodriguez Ortiz 1974)

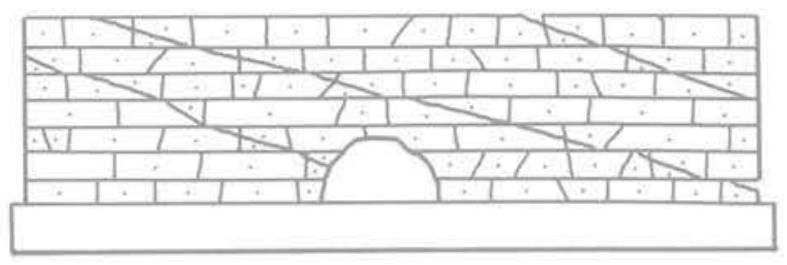

état initial

(angle de frottement

entre blocs $=30^{\circ} \mathrm{)}$

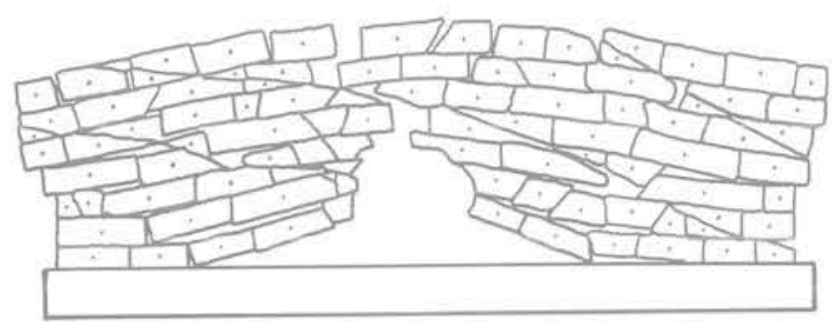

déplacement

vertical

maximum

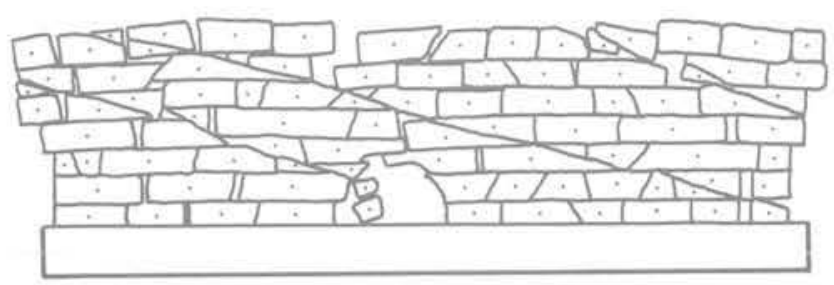

équilibre

final

Fig. 6 Déplacement des blocs surmontant une cavité soumise à une explosion (Cundall 1973) 


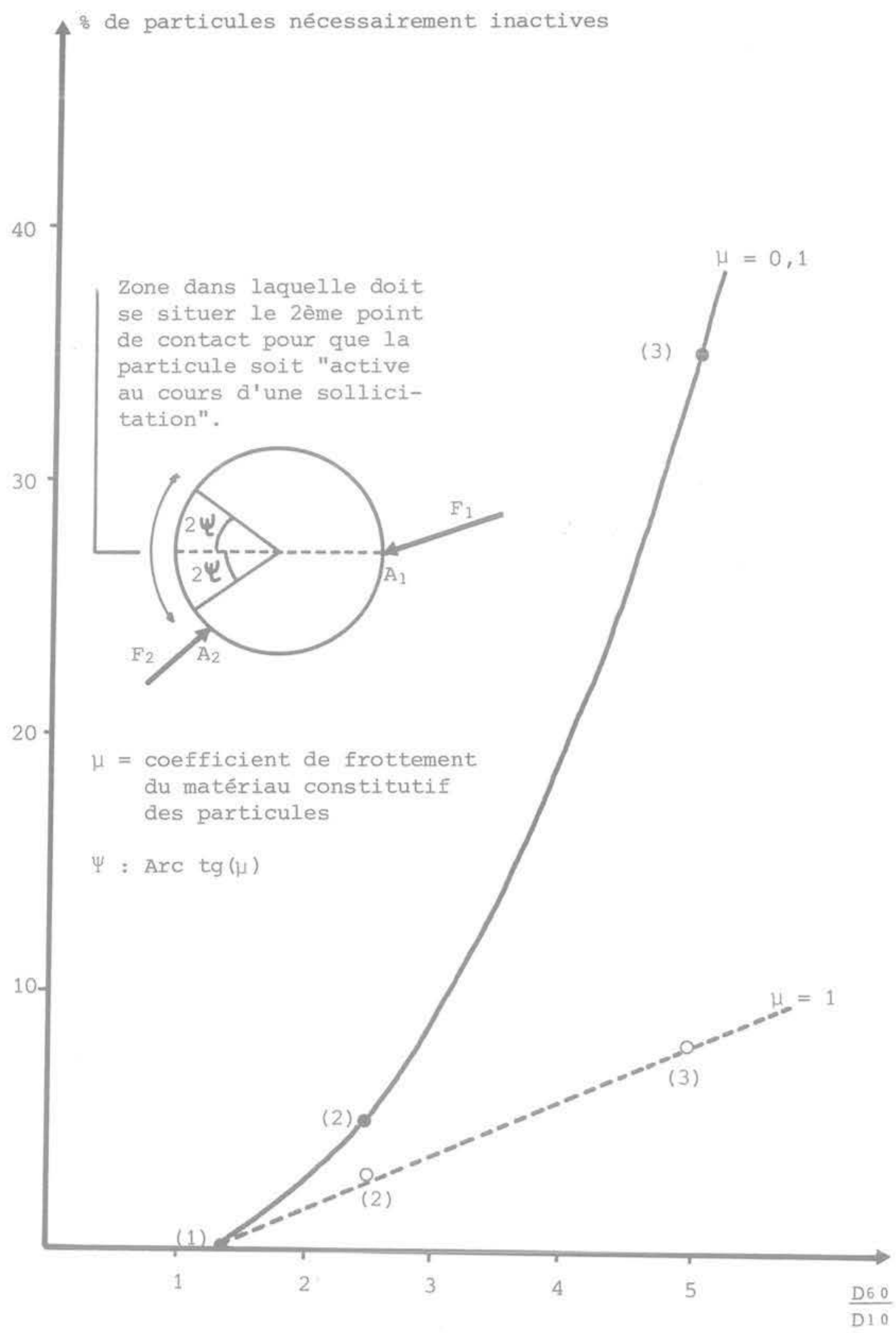

Fig. 7 Pourcentage de particules nécessairement inactives dans un matériau composé de 500 particules cylindriques simplement déversées en fonction de la granulométrie de ce matériau

Chaque matériau étudié est défini par une courbe granulométrique qui peut être quelconque et par le coefficient de frottement du matériau des particules. Ce matériau est déversé, sous l'action de la pesanteur dans un moule dont on a préalablement défini les dimensions. Le mode de déversement peut être modifié afin d'obtenir des milieux de différentes compacités.

Le programme réalise ensuite l'analyse statistique des diverses propriétés géométriques tant au niveau des particules que des arrangements élémentaires de particules.

- Nous avons pu montrer l'existence de particules nécessairement inactives pour toute sollicitation de la structure granulaire, leur nombre augmente avec l'étendue de la granulométrie et diminue avec l'angle de frottement du matériau des particules (fig. 7). 

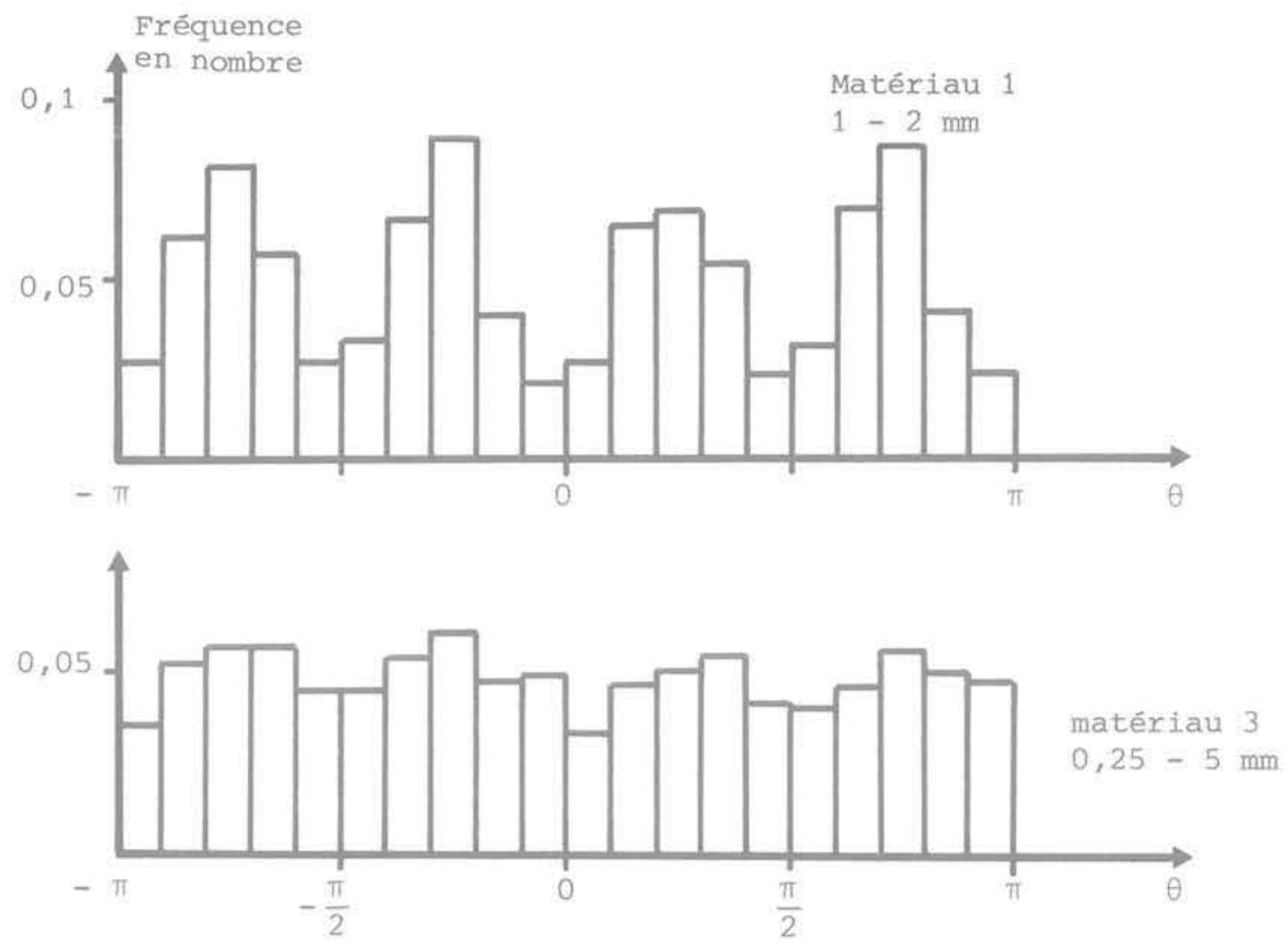

Fig. 8 Influence de la granulométrie sur l'histogramme de fréquence de l'orientation des rayons de contact (mesure de I'anisotropie)

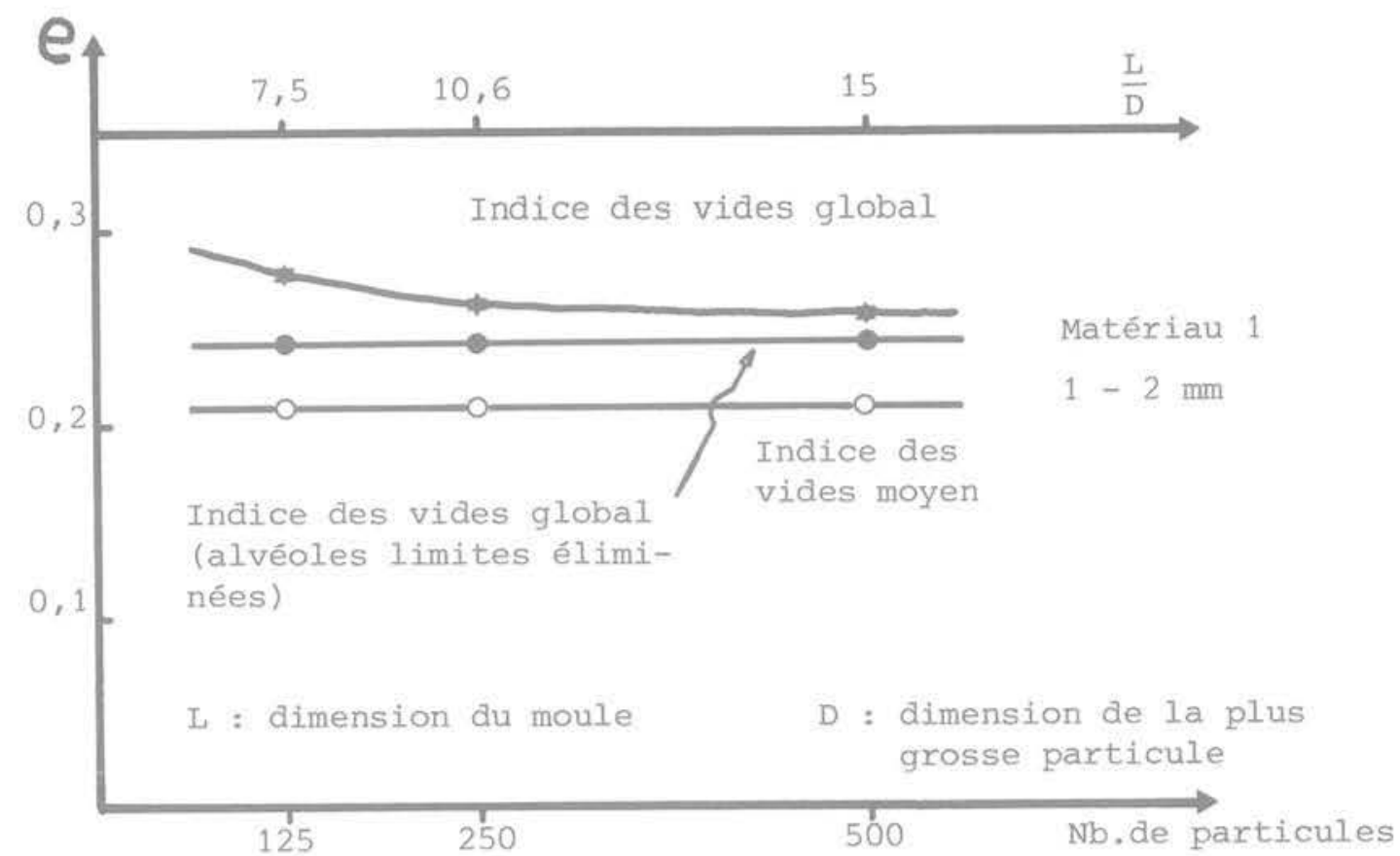

Fig. 9 Influence de la dimension du moule sur les mesures globales de compacité

- Notre étude confirme l'existence d'une anisotropie du milieu qui est plus élevée pour les matériaux de granulométrie serrée (fig. 8).

- Les distributions des différentes variables ponctuelles sont très sensibles à l'étendue de la granulométrie du matériau, en particulier l'indice des vides ponctuel (fig. 10).

- Pour un milieu bidimensionnel, les mesures globales de compacité ne seront représentatives que si la dimension du moule est supérieure à environ 10 fois la dimension de la plus grosse particule (fig. 9).

- Pour les types de matériaux bidimensionnels étudiés, la notion de milieu continu ne peut être utilisée que dans des domaines dont les dimensions sont au moins égales à 3 à 8 fois les dimensions de la plus grosse particule (fig. 11). 


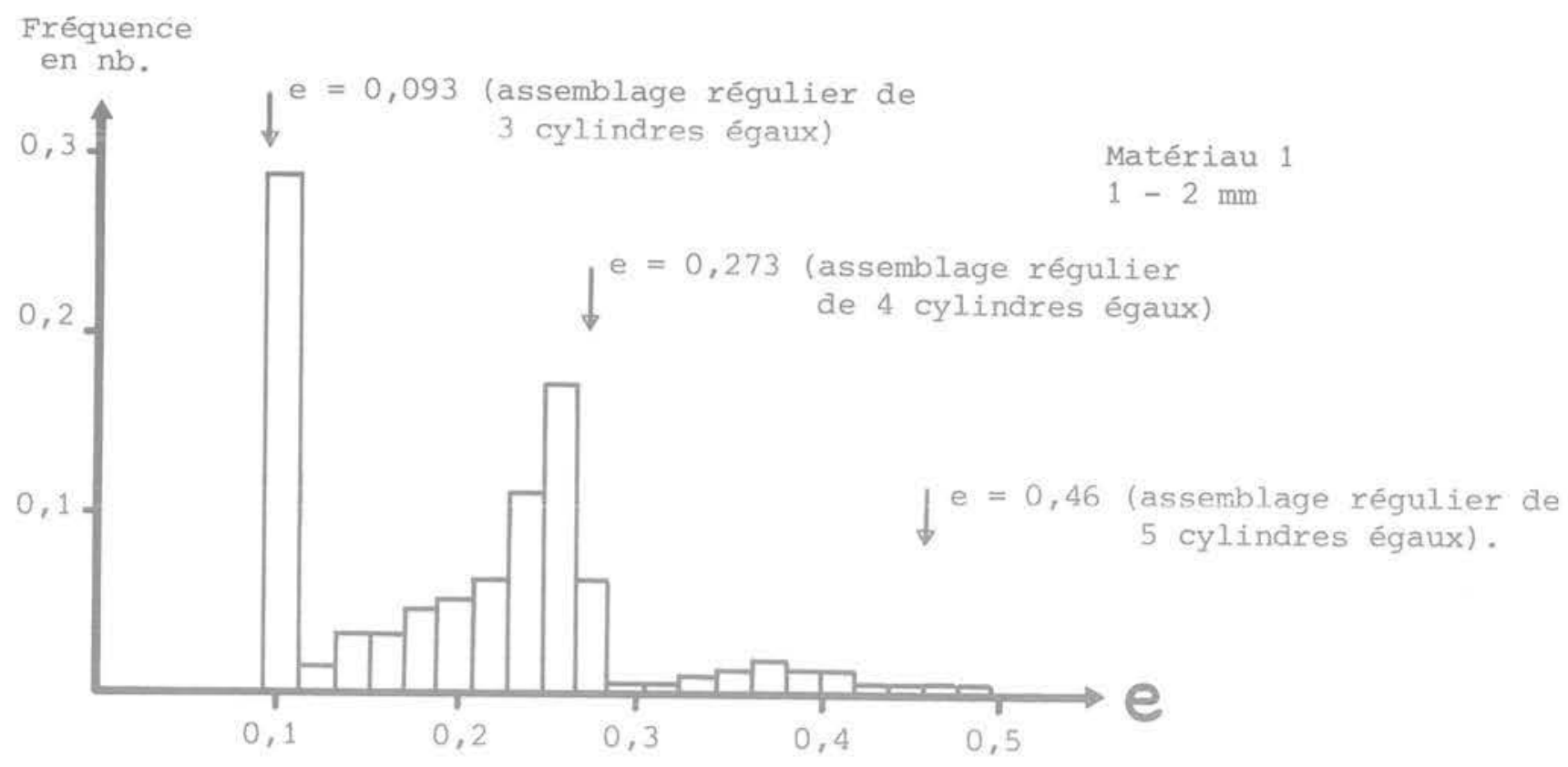

\section{Fréquence}

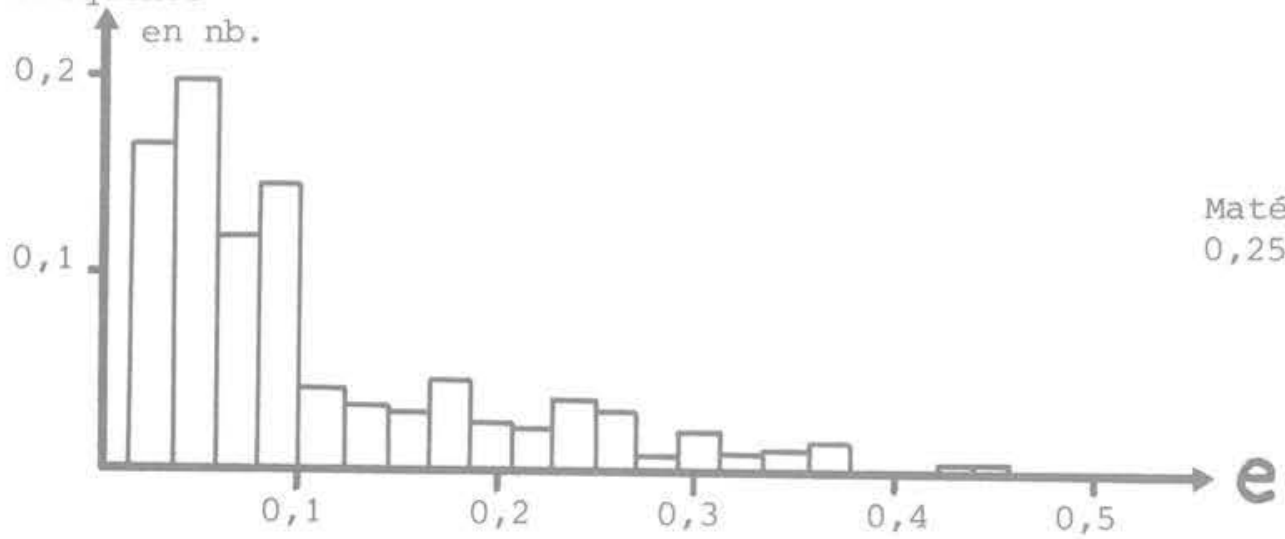

Fig. 10 Influence de la granulométrie sur l'histogramme de fréquence de l'indice des vides des arrangements élémentaires (milieu bidimensionnel)

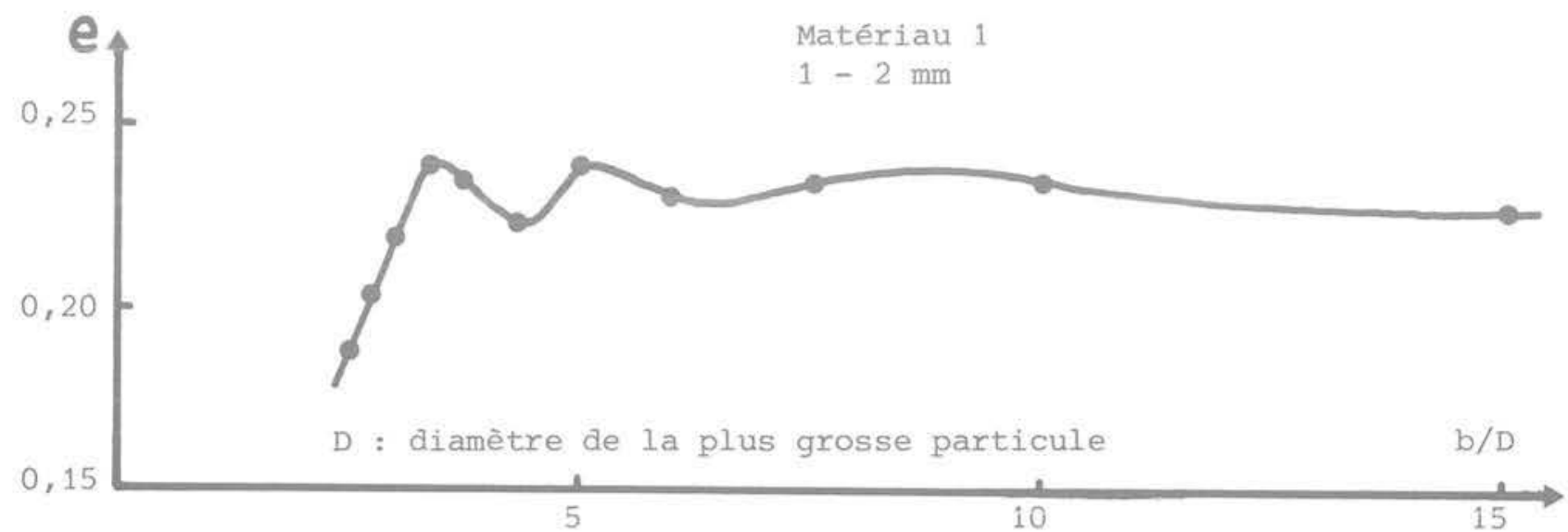

Fig. 11 Variation de l'indice des vides global en fonction du diamètre b du cercle à l'intérieur duquel s'effectue la mesure 


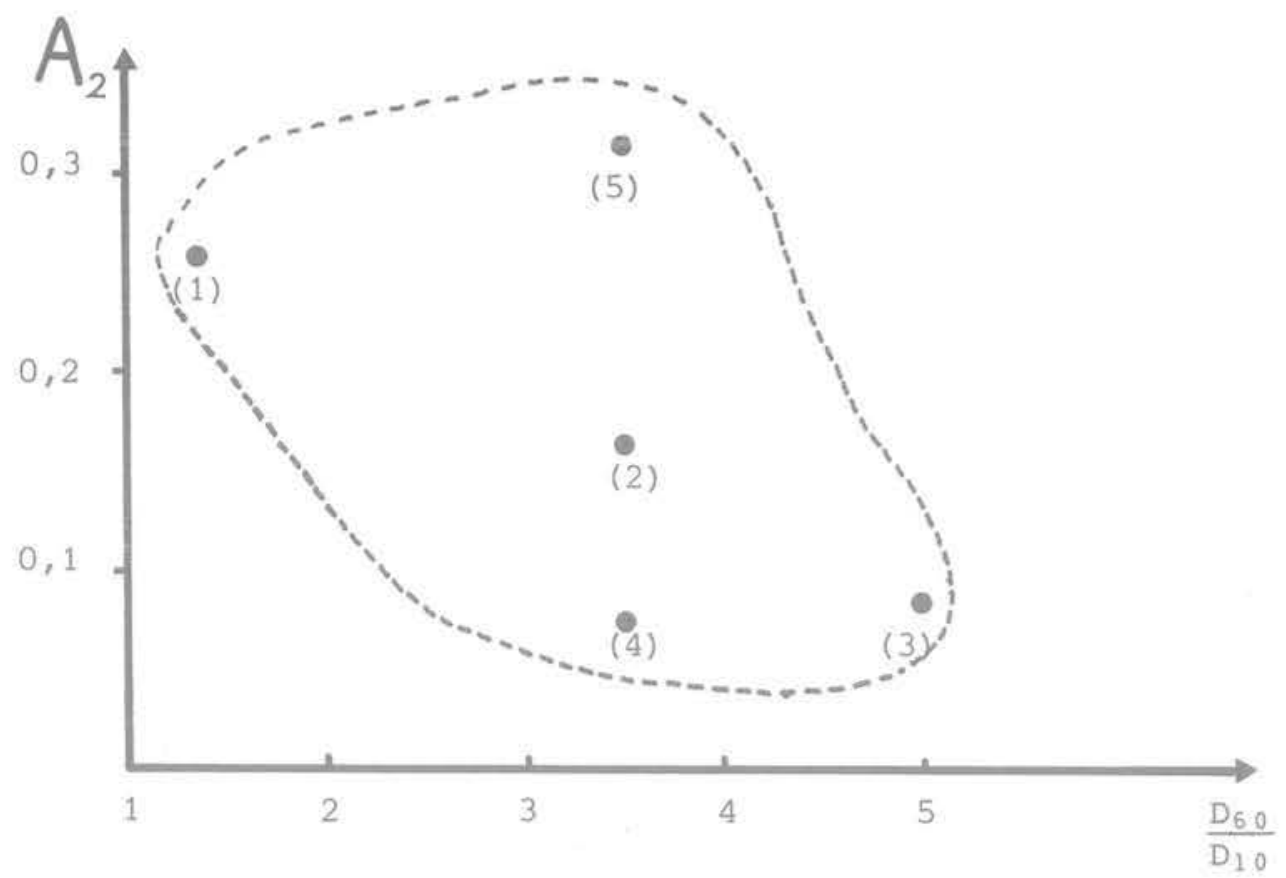

Fig. 12 Anisotropie des matériaux en fonction du coefficient $D_{60} / D_{10}$ relatif à la courbe granulométrique en poids

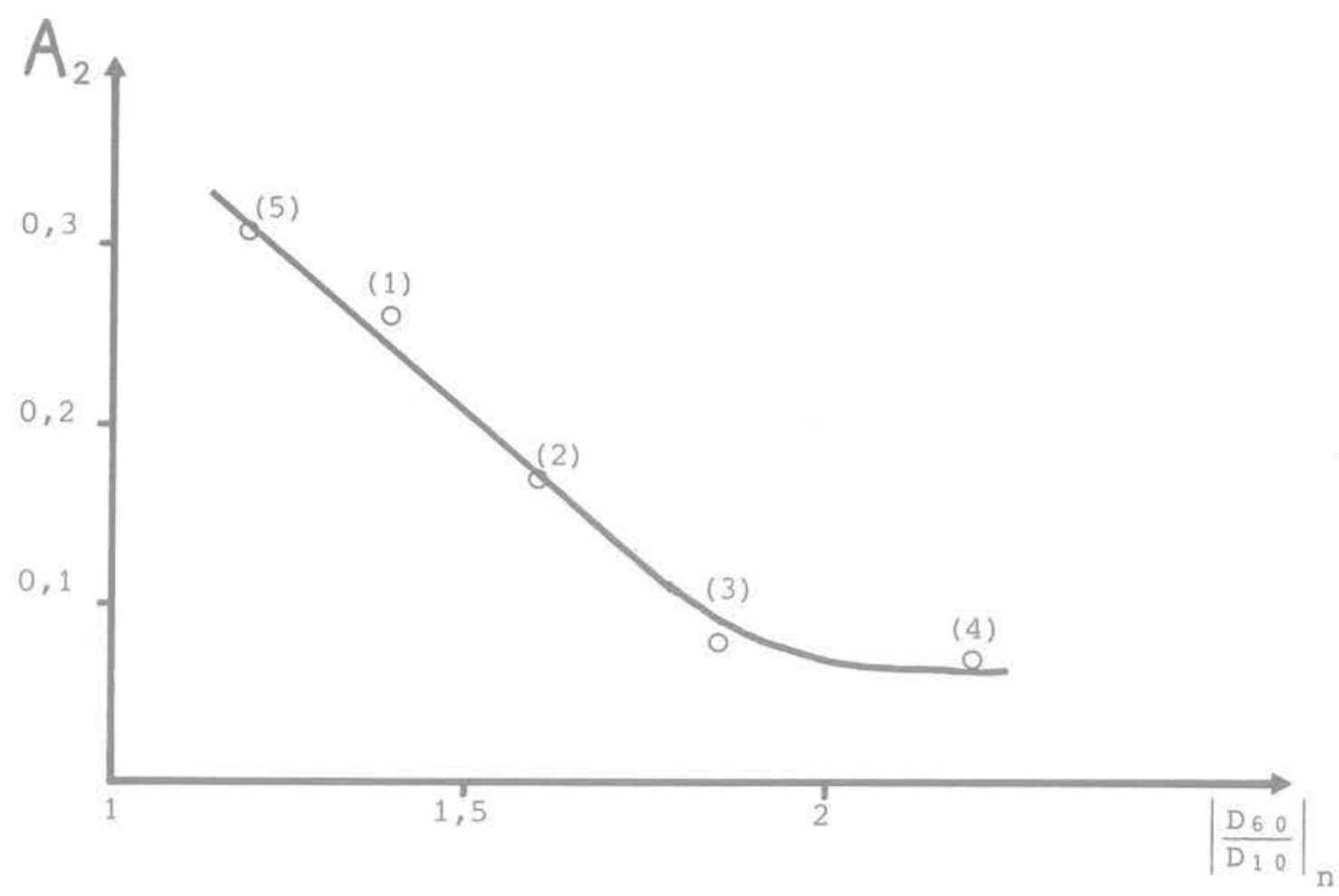

Fig. 13 Anisotropie des matériaux en fonction du coefficient $D_{60} / D_{10}$ relatif à la courbe granulométrique en nombre

- Le coefficient d'uniformité de la courbe granulométrique en poids ne semble pas être une très bonne caractéristique de la structure du matériau, car il ne prend pas en considération les très nombreuses particules de dimension $\angle D_{10}$. II semble que $\mathrm{D}_{60} / \mathrm{D}_{\min }$ ou encore $\left[\mathrm{D}_{80} / \mathrm{D}_{10}\right]_{\mathrm{n}}$ correspondant à la courbe granulométrique en nombre seraient des paramètres beaucoup plus représentatifs (fig. 12 et 13).

L'analyse des distributions des variables ponctuelles permet une première approche qualitative du comportement; ainsi l'on peut prévoir que le matériau de granulométrie étendue, simplement déversé, sera beaucoup plus déformable que celui de granulométrie serrée. En effet ce dernier possède plus de particules ayant un nombre de contacts élevé (plus de 4 contacts) donc, dont le déplacement sera plus difficile sous sollicitation et possède moins d'arrangements instables (comportant de nombreuses particules). Ce type d'analyse, qui reste très sommaire, est en bon accord avec l'expérience (fig. 14 et 15).

De même on peut prévoir que si le matériau de granulométrie étalée (comportant quelques arrangements alvéolaires) est soumis à des sollicitations rapides il y aura destruction des arrangements peu stables, donc variation de volume importante d'où risque de liquéfaction par augmentation de la pression interstitielle. Ce risque sera semble-t-il plus faible avec le matériau de granulométrie très serrée. 


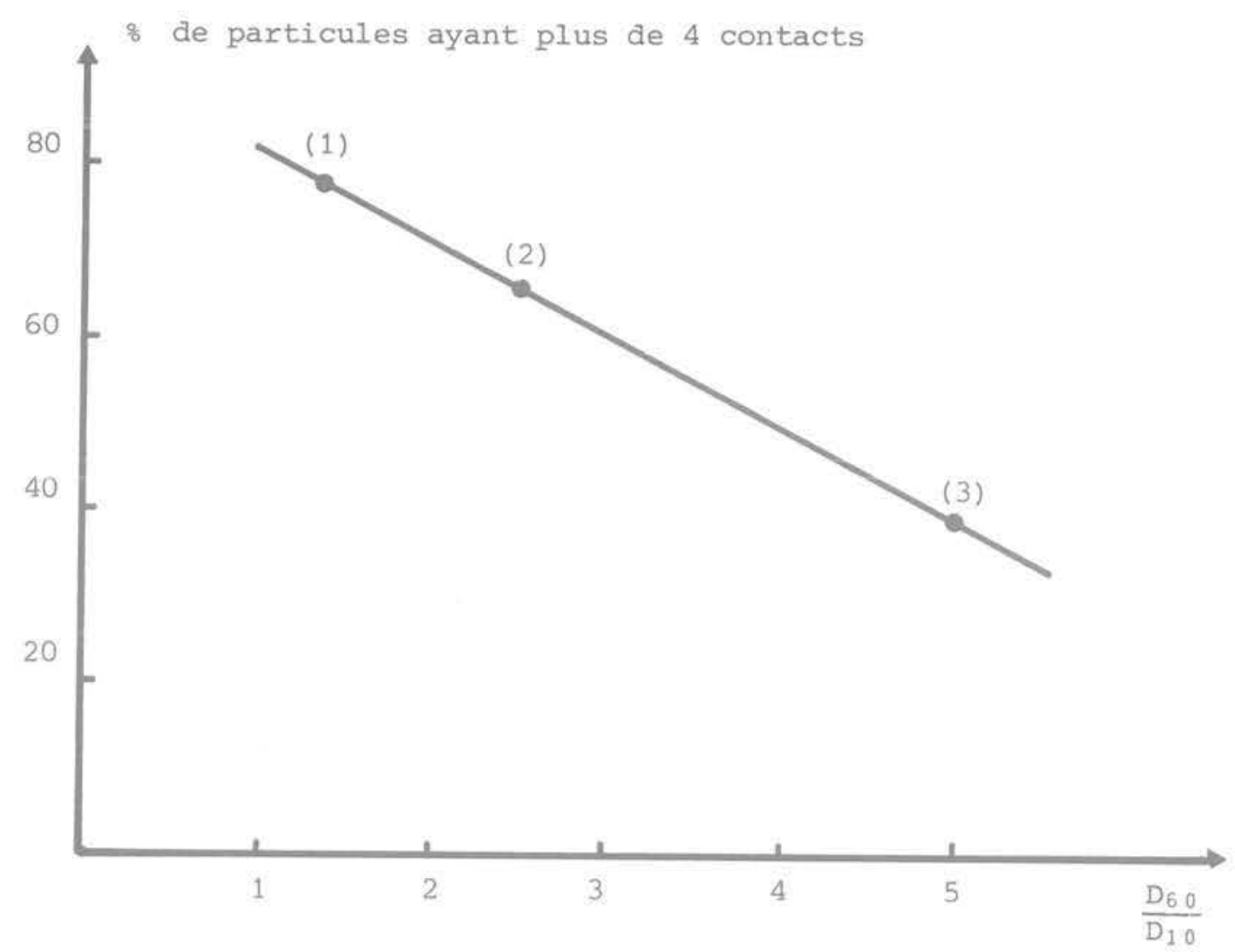

Fig. 14 Evolution du pourcentage de particules ayant plus de 4 contacts en fonction de la granulométrie (milieu bidimensionnel)

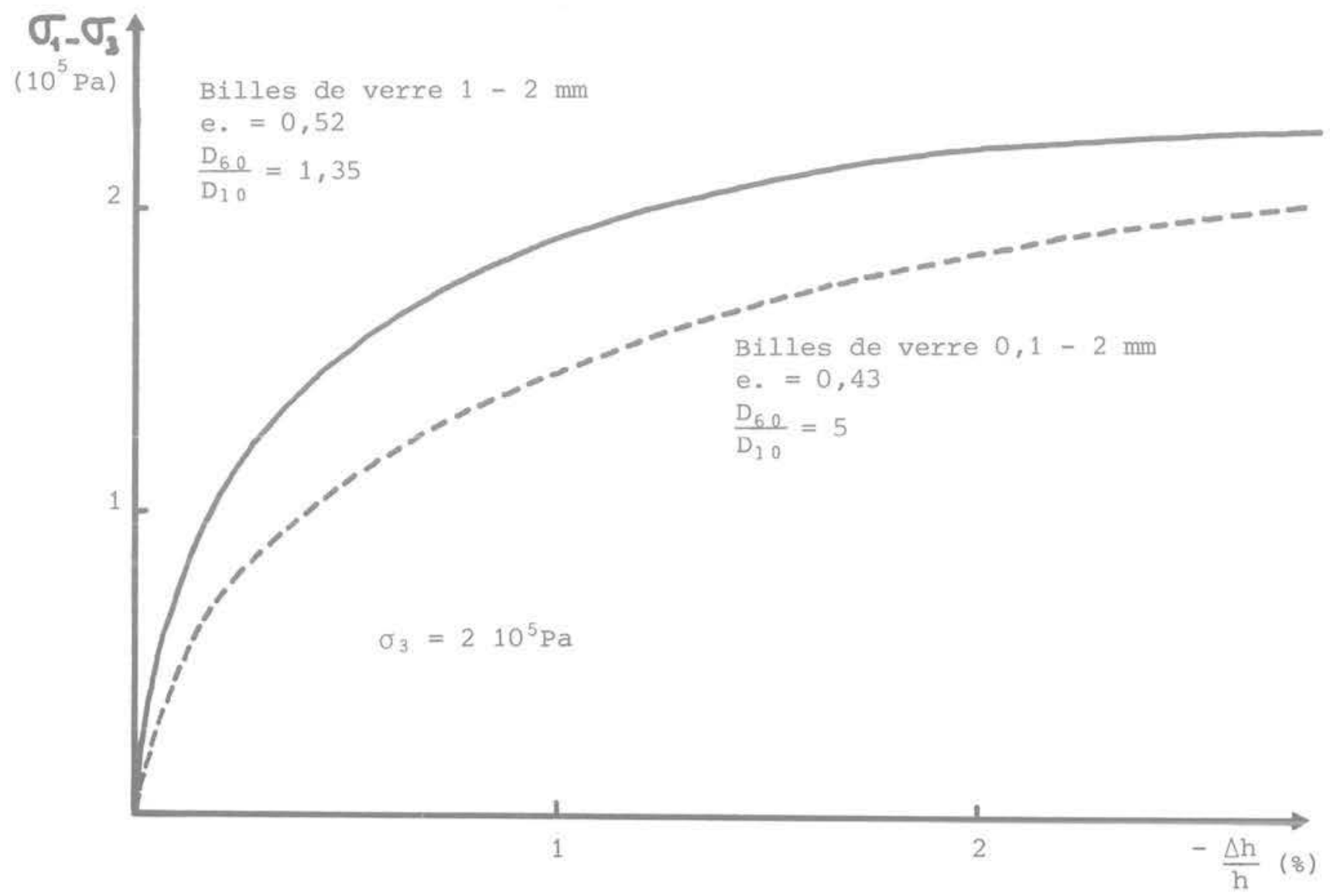

Fig. 15 Essai triaxial sur des billes de verre, simplement déversées 


\subsection{Modèle numérique de sollicitation d'un milieu granulaire}

La sollicitation est définie, en contraintes, sur les limites de l'échantillon construit à partir du programme précédemment décrit.

Nous cherchons à analyser le comportement ponctuel et global de l'échantillon, pour cela nous avons été amenés à faire un certain nombre d'hypothèses probabilistes en particulier sur la répartition des forces de contact dans la structure.

\subsubsection{Les forces de contact}

Soit $\vec{F}_{1}$ la force au contact $i$, ses composantes suivant les 3 axes de références sont $F_{i 1}, F_{i 2}, F_{i 3}$. Ces composantes dépendent des sollicitations appliquées et de la géométrie de la structure définie par un très grand nombre de paramètres. On peut donc considérer que chaque variable telle que $F_{i j}(j=1,2,3)$ appartient à un processus stochastique spatial défini par une distribution.

Une étude menée par la méthode des éléments finis sur un milieu continu hétérogène a confirmé cette hypothèse et a permis de conclure que la distribution des contraintes suivant les deux directions des sollicitations extérieures est de forme normale et admet un coefficient de variation qui semble constant au cours d'une sollicitation monotone.

Ces conclusions ont été reprises en hypothèses pour les distributions des variables $F_{i j}(j=1,2,3)$.

$\mathrm{Si}$ la sollicitation extérieure est définie par les contraintes principales $\sigma_{1}, \sigma_{2}, \sigma_{3}$, on montre que les moyennes des variables $F_{i j \theta}$ (composante $j$ de la force du contact i orienté de $\theta$ ) (Cambou 1979) sont égales à (fig. 16) :

$$
\left|\overline{F_{11 \theta}}\right|=\frac{\pi \sigma_{1} S_{1} \sin \theta}{n_{1} \int_{0}^{\pi} f(\theta) \sin \theta d \theta}
$$

$\mathrm{S}_{1}$ est la surface d'un plan perpendiculaire à $\sigma_{1}$ qui coupe $n_{1}$ particules.

$$
\begin{gathered}
\left|\bar{F}_{12 \theta}\right|=\frac{\pi^{2} \sigma^{2} S^{2}|\cos \theta \sin \alpha|}{2 n_{2} \int_{0}^{\pi / 2} \int_{0}^{\pi} f(\alpha) f(\theta)|\cos \theta \sin \alpha| d \theta d \alpha} \\
\left|\bar{F}_{i 3 \theta}\right|=\frac{\pi^{2} \sigma_{3} S_{3}|\cos \theta \cos \alpha|}{2 n_{3} \int_{0}^{\pi / 2} \int_{-\pi / 2}^{\pi / 2} f(\alpha) f(\theta)|\cos \theta \cos \alpha| d \theta d \alpha} .
\end{gathered}
$$

La distribution des variables telles que $F_{i j \theta}$ est ainsi entièrement définie par sa moyenne, sa forme (normale) et son écart type (fixé à partir de résultats expérimentaux sur des particules photoélastiques par exemple).

Chaque force de contact est donc déterminée par tirage au sort dans les distributions ainsi définies des trois valeurs $F_{i j e}(j=1,2,3)$.

\subsubsection{Les déformations}

Les déformations dans le milieu sont dues:

- aux glissements entre particules

- aux déformations des particules

- aux ruptures de particules.

Nous ne prendrons en considération ici que les deux premiers types de déformations, prépondérants pour des états de contrainte pas trop élevés.

\section{- Déformations dues aux glissements entre particules}

- Glissement entre deux particules

II y a glissement si l'angle $\rho_{1}$ entre la force de contact et la normale est supérieur à $\Psi$, angle de frottement du matériau des particules, donc pour:

$\cos \Psi \geqslant \sin \beta_{1} \cos \alpha_{i} F_{13}+\sin \beta_{i} \sin \alpha_{i} F_{12}+\cos \beta_{i} F_{i 1}$.

La direction de glissement définie par les angles $\delta_{i}, \gamma_{i}$ peut être déterminée analytiquement en fonction de la force de contact et de l'orientation du contact (Cambou 1979).

Le module du vecteur glissement est a priori inconnu, nous avons proposé une expression de la forme :

$$
\left|\Delta \vec{U}_{i}\right|=a\left(e_{i}+b\right) \frac{T_{i}}{N_{i}} \frac{\left|\vec{F}_{i}\right|}{F_{M}} \frac{N_{g}}{\left(N_{p}-N_{g}\right)}
$$

$e_{1}$ : indice des vides dans le voisinage du point $i_{1}$

$N_{1}$ : composante normale de $\vec{F}_{i}$,

$T_{1}$ : composante tangentielle de $F_{i}$,

$\mathrm{N}_{\mathrm{p}}$ : nombre de contacts glissants entraînant la mise en plasticité,

$\mathrm{N}_{\mathrm{g}}$ : nombre de contacts glissants,

$F_{M}$ : valeur moyenne du module des forces de contact.

Ce module de glissement est défini à une constante près, a, que l'on détermine à partir de la mesure des déformations globales sur une expérience particulière.

Cette expression tente de traduire un certain nombre de notions "intuitives", à savoir que le déplacement augmente quand :

- La force de contact s'éloigne du cône de frottement $\left(\frac{T_{i}}{N_{i}}\right)$.

- La force de contact est élevée $\left(\frac{\vec{F}_{1}}{F_{M}}\right)$.

- Le milieu est peu compact au voisinage $\left(e_{1}+b\right)$.

- Le nombre de contacts glissants dans le milieu est élevé $\left(\frac{N_{p}}{N_{p}-N_{g}}\right)$.

- $\left|\Delta \vec{U}_{i}\right|$ sera infini pour $N_{g}=N_{p}$, ce qui se traduit par la mise en plasticité globale du milieu.

Soit $\mathrm{N}_{\mathrm{e}}$ le nombre de contacts assurant statistiquement un équilibre isostatique de la structure. Ce nombre est déterminé numériquement en analysant l'équilibre de chaque particule. Si le nombre de contacts glissants $\mathrm{N}_{\mathrm{o}}$ est égal à $\left(\mathrm{N}-\mathrm{N}_{\mathrm{e}}\right)$, tout nouveau contact glissant détruit l'équilibre global de la structure, donc $\mathrm{N}_{\mathrm{p}}=\mathrm{N}-$ $N_{\text {e }}$. Ce type de calcul suppose que la répartition des contacts glissants est homogène dans toute la structure. La structure étant suffisamment « souple * pour autoriser les déplacements internes, cette hypothèse semble correcte.

\section{- Déformation globale due aux glissements:}

On construit un chemin entièrement solide à travers l'ensemble granulaire formé de particules sphériques (Oda 1974, Horne 1970) (fig. 17). Pour $\Delta z=1$, le nombre de particules interceptées est égale à $m_{1 z}=\frac{1}{2|\overline{R \sin \theta}|}$

Si $p$ est le pourcentage de contacts glissants, le déplacement de la particule $m_{1 z}$ sera égal à : 


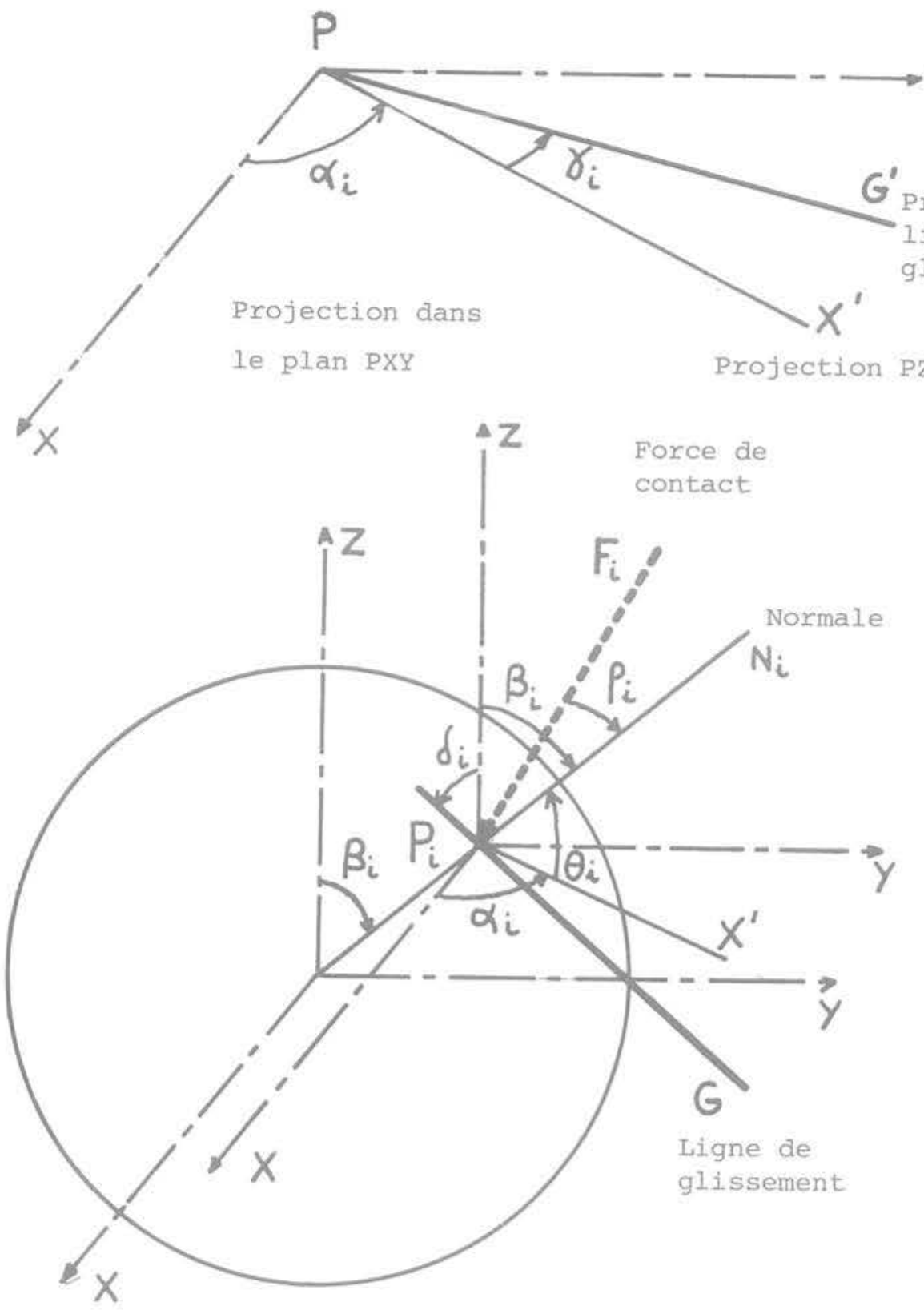

Fig. 16 Définition des variables caractérisant le point de contact $P_{i}$ entre deux particules

$$
\mathrm{u}_{z}=\sum_{i=1}^{1=\mathrm{pm}_{i z}} \mathrm{~S}_{\mathrm{iz}}\left|\Delta \overrightarrow{\mathrm{U}}_{i}\right|\left|\cos \delta_{i}\right|
$$

$S_{i z}= \pm 1$ suivant que le déplacement correspond à un aliongement ou un raccourcissement dans la direction Z.

$$
\begin{aligned}
\varepsilon_{z}=\frac{\delta u_{z}}{\delta_{z}} & =\frac{m_{1 z}}{N} \sum_{i=1}^{i=p N} S_{i z}\left|\Delta \vec{U}_{i}\right|\left|\cos \delta_{i}\right| \\
\varepsilon_{x} & =\frac{m_{1 x}}{N} \sum_{i=1}^{i=p N} S_{i x}\left|\Delta \vec{U}_{i}\right|\left|\sin \delta_{i} \cos \gamma_{i}\right| \\
\varepsilon_{y} & =\frac{m_{1 y}}{N} \sum_{i=1}^{i=p N} S_{i y}\left|\Delta \vec{U}_{i}\right|\left|\sin \delta_{i} \sin \gamma_{i}\right| .
\end{aligned}
$$

- Déformations dues aux déformations des particules Les déformations aux contacts entre deux particules peuvent être calculées par les formules proposées par Hertz et Mindlin (Duffy, Mindlin 1957).

A partir des chemins solides définis précédemment, on détermine aisément les déformations globales (Cambou 1979).

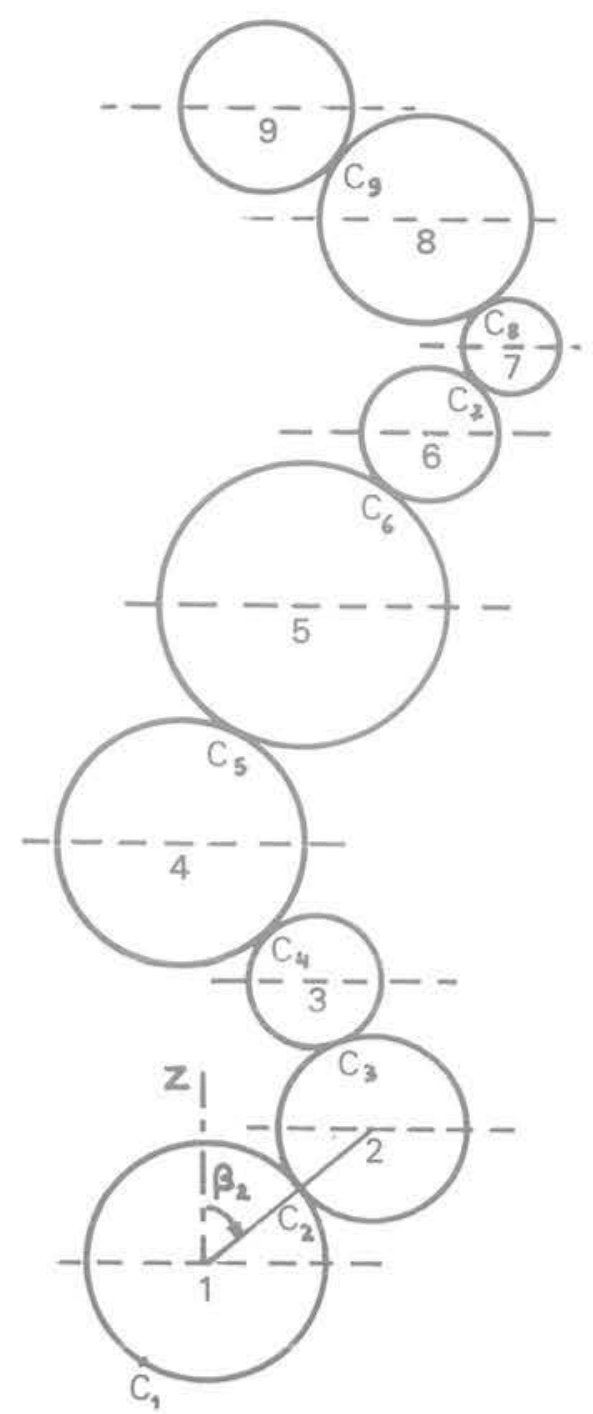

Fig. 17 Chemin solide de direction générale OZ à travers une structure granulaire 


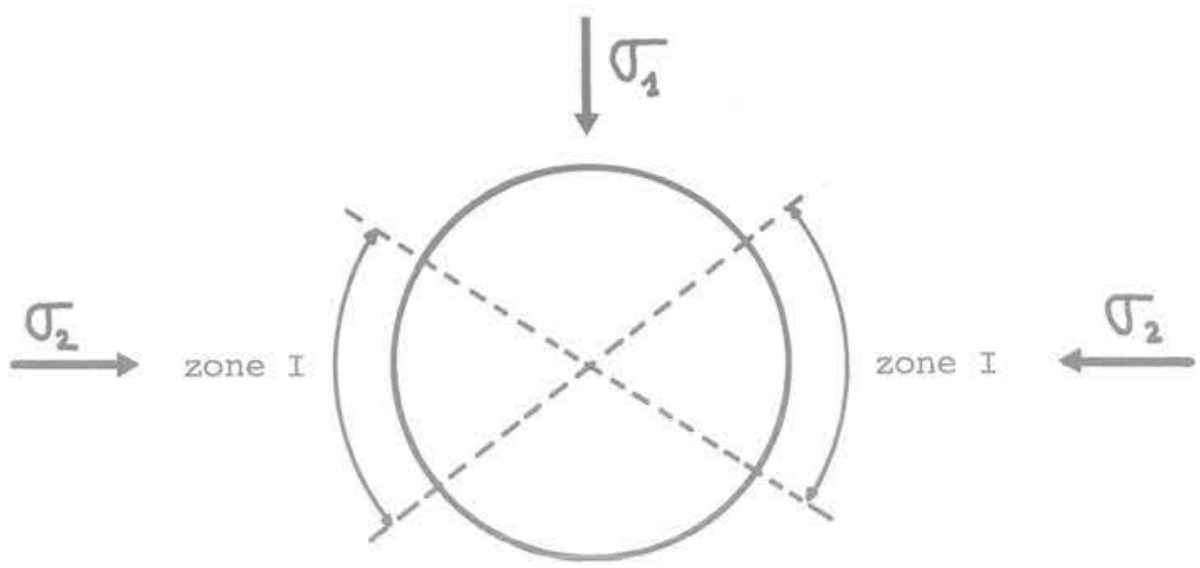

Fig. 18 Modèle statique, orientation des contacts glissants (zone I)

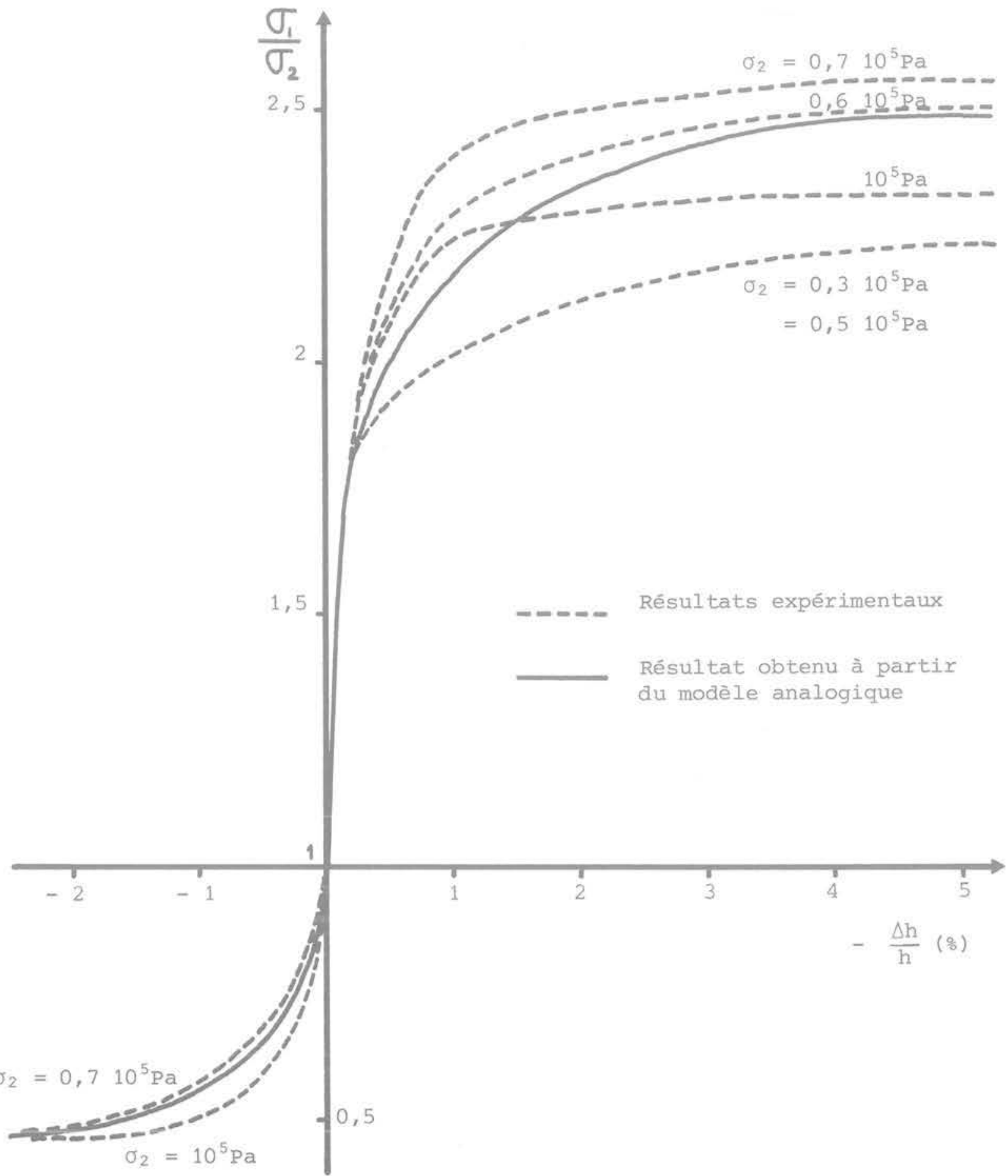

Fig. 19 Comparaison entre résultat du modèle analogique (mat. $1-2 \mathrm{~mm}$ ) et résultats expérimentaux sur un milieu formé de petits rouleaux en duralumin $(3$ et $5 \mathrm{~mm})$. Sollicitation biaxiale 


\subsubsection{Prise en considération de conditions cinématiques}

Le modèle défini précédemment est basé sur une analyse statique (équilibre des forces dans la structure). Chaque contact est traité séparément, or, dans la réalité les déplacements des particules les unes par rapport aux autres sont régis par des conditions cinématiques (compatibilité des déplacements).

Une première étude uniquement statique, pour une sollicitation non isotrope, a permis de noter que les contacts glissants se localisent dans une zone d'orientation bien définie (zone l, fig. 18).

En fait pour assurer la compatibilité des déplacements (conditions cinématiques) il se produira nécessairement des glissements hors de la zone I; le nombre de ces contacts est essentiellement lié aux déformations, i) sera maximum en grandes déformations (mise en plasticité).

Le modèle statique a donc été modifié en imposant un certain nombre de contacts glissants hors de la zone (nombre lié à l'état de déformation).

\subsubsection{Le programme de calcul}

Le programme permet de déterminer numériquement pour chaque incrément de sollicitation en contrainte les déformations principales.

Pour un incrément de sollicitation donné, on passe en revue tous les points de contact, si il y a glissement en un point on détermine la composante qui l'a provoqué, soit $F_{i 1}$ par exemple, $F_{11}>F_{i 1 m}$ (correspondant à la limite du cône de frottement).

On ramène $F_{i 1}$ à $F_{i 1 m}$, il faut donc reporter $\left(F_{i 1}-F_{i 1 m}\right)$ sur un autre contact. Ce report se fait sur des contacts n'appartenant pas à la zone d'orientation I de façon à introduire les conditions cinématiques.

On effectue plusieurs boucles sur tous les contacts jusqu'à obtenir l'équilibre global. On s'assure en outre que le nombre de contacts glissants situés hors de la zone I est suffisant pour répondre aux conditions cinématiques.

Les déformations par glissement sont calculées au cours de ces diverses boucles, la dernière boucle étant utilisée pour déterminer les déformations dues aux déformations des particules.

\subsubsection{Analyse des résultats}

Les résultats présentés se rapportent à des milieux bidimensionnels et tridimensionnels. Pour une simulation d'essai biaxial on observe des résultats semblables à ceux obtenus expérimentalement (fig. 19).

Les courbes intrinsèques sont des droites et la courbe $\sigma_{1} / \sigma_{3}=f\left(\varepsilon_{1}\right)$ est la même quelle que soit la valeur de $\sigma_{3}$.

On notera que les valeurs des déformations sont calculées à une constante multiplicative près qui a été déterminée de façon à approcher au mieux la courbe expérimentale.

- Comparaison entre la distribution des déformations dans le milieu granulaire et la distribution tensorielle dans le milieu continu.

Nous avons calculé numériquement les dilatations suivant différentes orientations $\omega$.

Pour une sollicitation dont les axes principaux sont confondus avec les axes de symétrie de l'anisotropie géométrique structurelle due à la pesanteur, les valeurs calculées pour le milieu granulaire et pour le milieu continu sont très proches (fig. 20).

Ceci nous conduit à deux types de conclusion :

a) Cela justifie l'utilisation de la mécanique des milieux continus dans le cas des milieux granulaires.

b) Cela permet de penser que notre modèle de glissement est correct.

Pour une sollicitation en contrainte dont les axes principaux ne sont pas confondus avec les axes de symétrie de l'anisotropie géométrique, les directions principales des déformations sont confondues avec les axes de l'anisotropie géométrique au début de la sollicitation déviatoire puis elles évoluent pour atteindre l'orientation des directions principales du tenseur contrainte.

- Analyse des phénomènes ponctuels au cours de la sollicitation

\section{- Forces de contact}

On observe un maximum très marqué des modules des forces de contact dans la direction de la contrainte principale majeure (fig. 21),

Leur orientation par rapport à la normale présente une distribution de forme normale pour une sollicitation isotrope, elle évolue pour une sollicitation déviatoire et admet une valeur maximale égale à $\Psi$.

- Contacts glissants

Pour une sollicitation isotrope on observe un pourcentage de contacts glissants non négligeable, la distribution de leur orientation est alors sensiblement identique à celle des orientations de contact.

Pour une petite sollicitation déviatoire on observe une discontinuité très nette aussi bien en ce qui concerne le nombre de contacts glissants que leurs orientations (fig. 22, 23).

Soit $\beta_{m}$ la moyenne des $\left|\beta_{i}\right|$ correspondant à des contacts glissants, $\beta_{m}$ évolue au cours d'une sollicitation déviatoire (fig. 23).

- Analyse du comportement global à partir des phénoménes ponctuels

\section{- Anisotropie}

Sous l'action d'une sollicitation isotrope le milieu se déforme de façon anisotrope, ce qui traduit l'existence d'une distribution non uniforme des orientations de contact due à la mise en place dans le champ de la pesanteur.

\section{- Irréversibilité, écrouissage}

Lors du déchargement on peut constater que les déformations sont essentiellement irréversibles.

Le module en extension après une première compression est plus faible que lors d'une première sollicitation. II ne semble pas que cet écrouissage soit dû à une évolution de la géométrie de la structure (très faible avant l'écoulement plastique), mais plutôt au changement d'orientation des forces de contact au cours de la sollicitation (fig. 24).

On peut penser que, au cours de l'écoulement plastique les distributions des orientations de contact vont évoluer et provoquer un deuxième type d'écrouissage (notre modèle ne peut suivre l'évolution de la structure après le pic de plasticité et donc ne peut mettre en évidence ce phénomène). 


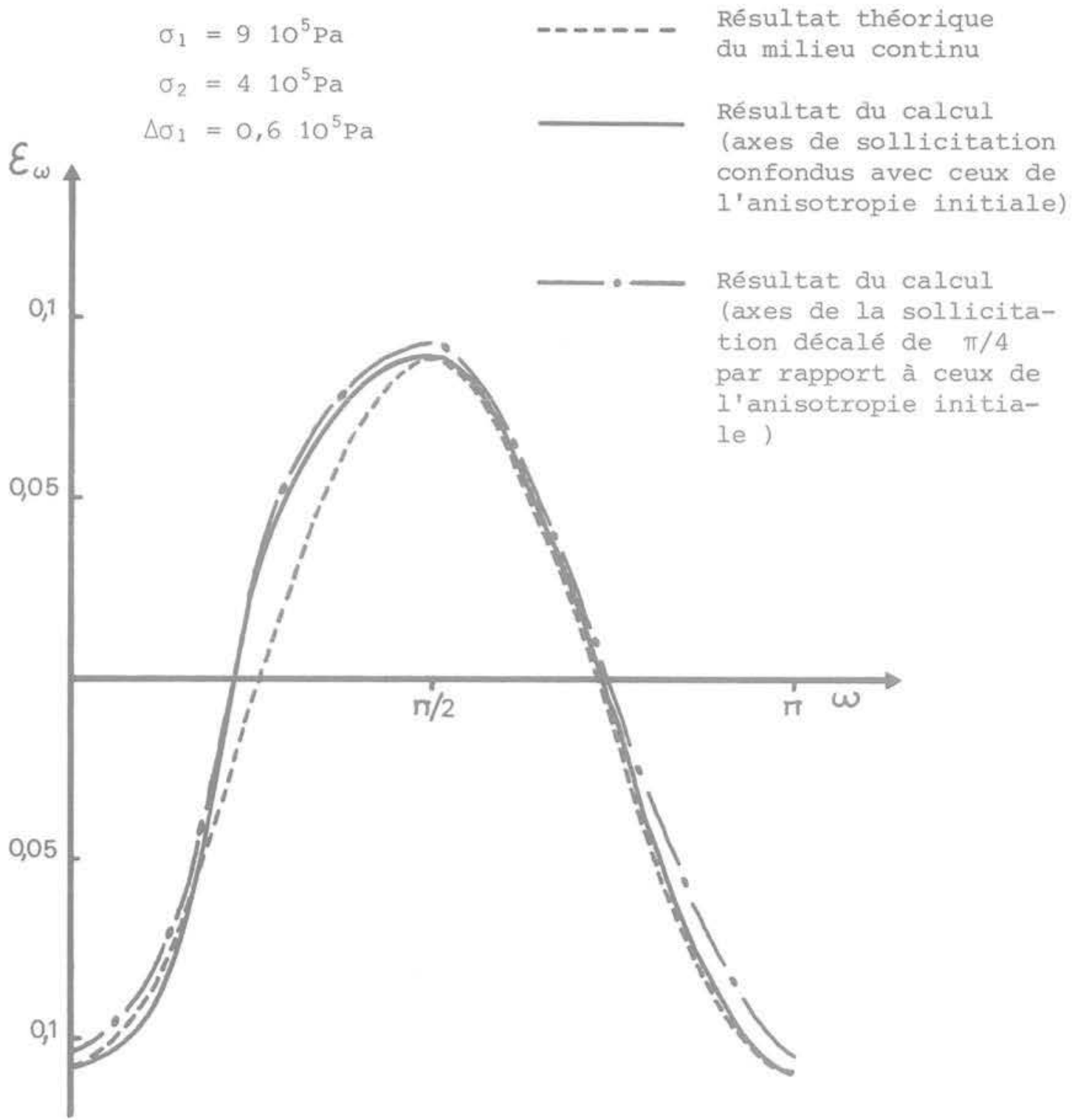

Fig. 20 Dilatations en fonction de l'orientation wconsidérée. Résultats obtenus par calcul numérique sur le modèle analogique. Matériau bidimensionnel simplement déversé $(1-2 \mathrm{~mm})$

- Dilatance

On ne prend en considération ici que les déformations dues aux glissements qui, pour une sollicitation déviatoire, sont en général prépondérantes.

Pour chaque incrément de déformation dû au glissement du contact $\mathrm{i}$ on $\mathrm{a}$, dans le cas d'une sollicitation bidimensionnelle :

$$
\left|\frac{\varepsilon_{i z}}{\varepsilon_{i x}}\right|=\frac{m_{1 z}}{m_{1 z}} \operatorname{tg} \beta_{i}
$$

On considère que, en première approximation tout se passe comme si tous les contacts glissants avaient une même orientation, égale à l'orientation moyenne $\beta_{m}$ (moyenne des $\left|\beta_{i}\right|$ des contacts glissants).

$\frac{m_{1 z}}{m_{1 x}}$ a été défini précédemment, il dépend de
I'anisotropie du matériau, nous avons posé $\frac{m_{1 z}}{m_{1 x}}=$ $\operatorname{tg}\left(\beta_{m}\right)_{0}$.

Le matériau sera dilatant pour $\beta_{m}<\left(\beta_{m}\right)_{0}$ et contractant pour $\beta_{m}>\left(\beta_{m}\right)_{0}$.

$\left(\beta_{m}\right)_{0}=45^{\circ}$ pour un matériau isotrope bidimensionnel, $\left(\beta_{m}\right)_{0}=52^{\circ}$ pour un matériau isotrope tridimensionnel soumis à une sollicitation axisymétrique (triaxial).

Pour une sollicitation en compression, dès l'apparition d'une petite contrainte déviatoire $\beta_{m}$ subit une discontinuité et I'on observe $\beta_{m}=58^{\circ}$ correspondant à un comportement contractant, au cours de la sollicitation $\beta_{m}$ diminue jusqu'à des valeurs inférieures à $\left(\beta_{m}\right)_{0}$ lorsqu'on approche du seuil de plasticité, le matériau présente alors un comportement dilatant ce qui est bien conforme aux résultats obtenus ainsi qu'aux résultats expérimentaux habituels (fig. 23). 

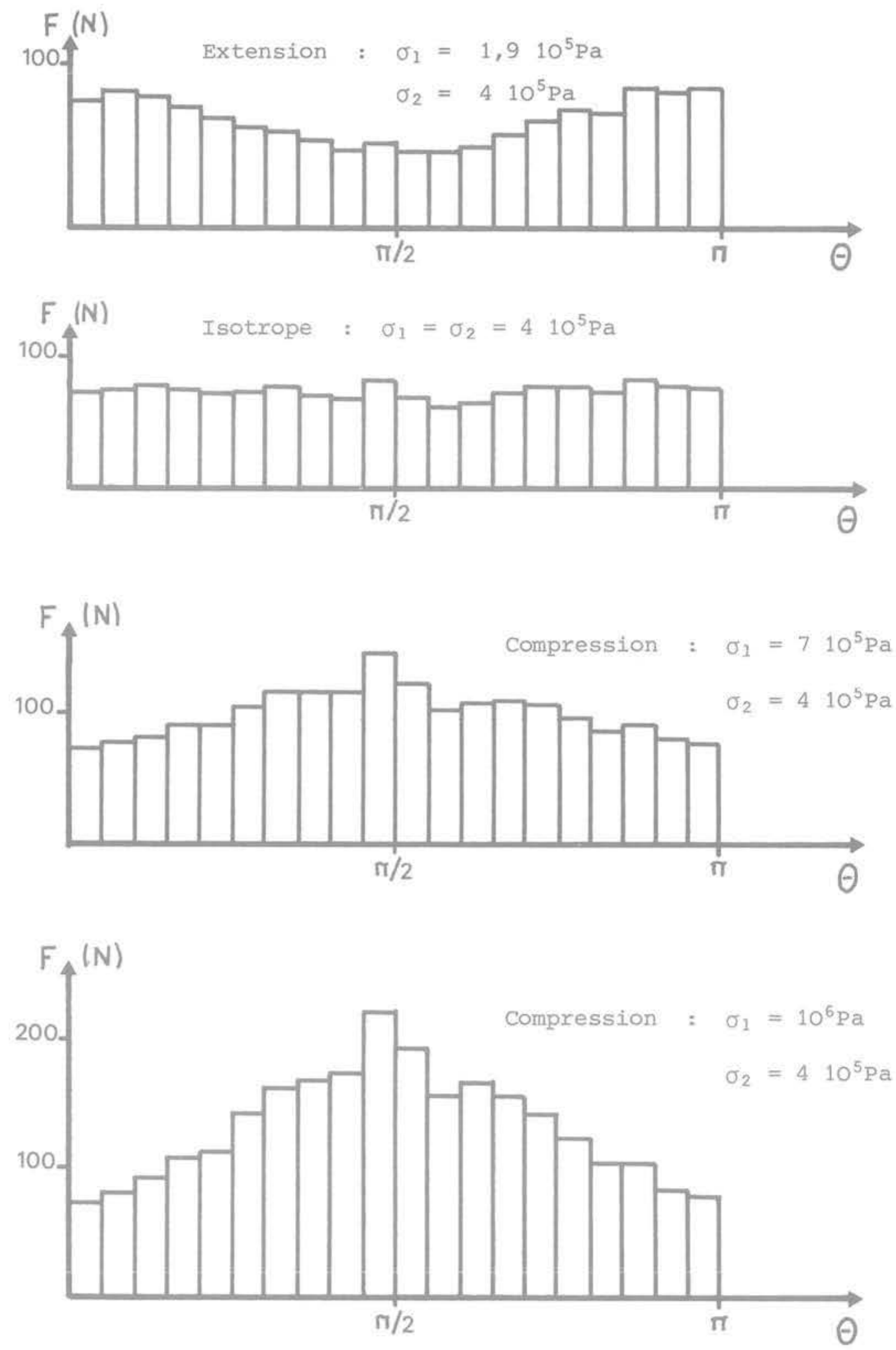

Fig. 21 Evolution du module moyen des forces de contact, au cours d'une sollicitation biaxiale. Résultats obtenus par calcul numérique sur le modẻle analogique. Matériau simplement déversé (1 $-2 \mathrm{~mm})$ 


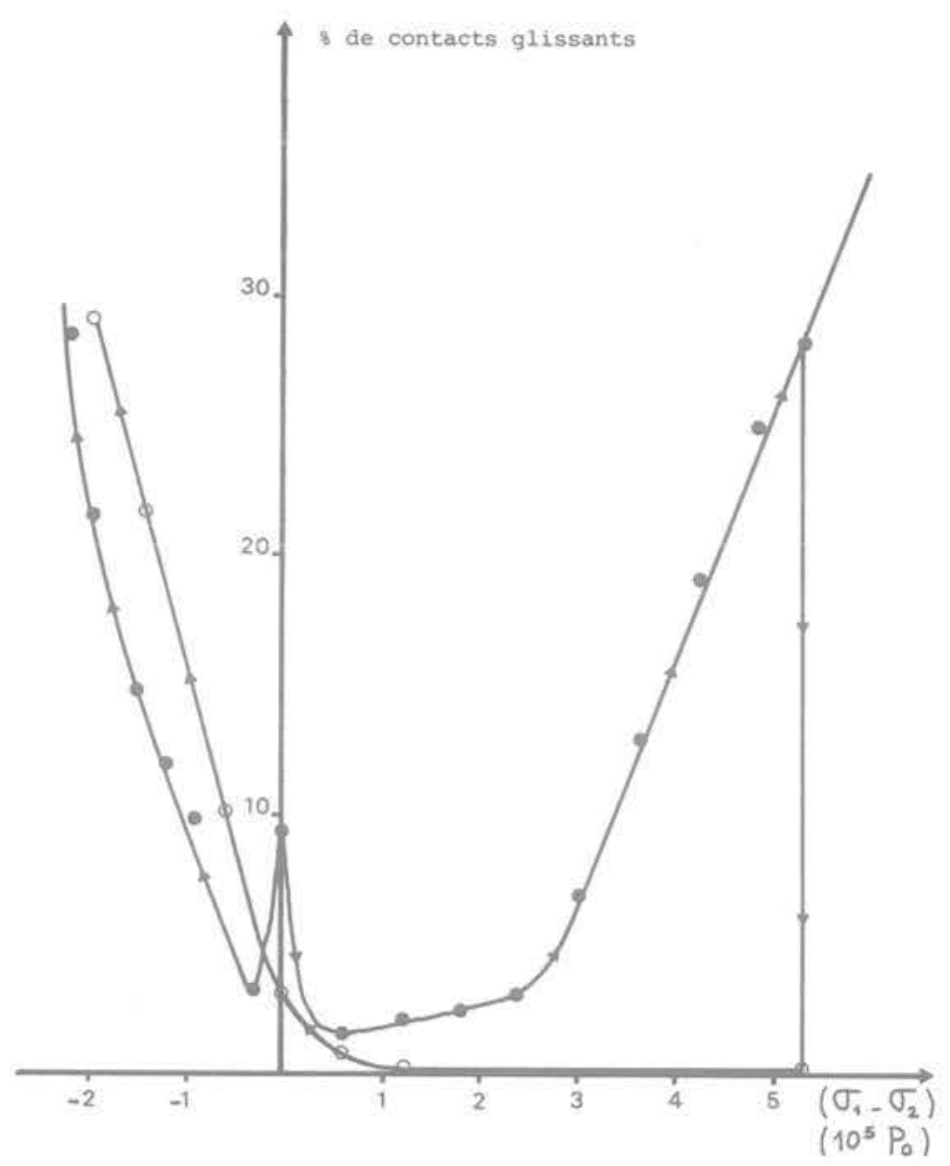

Fig. 22 Evolution du pourcentage de contacts glissants au cours d'une sollicitation biaxiale. Résultats obtenus par calcul numérique sur le modèle analogique. Matériau simplement déversé $(1-2 \mathrm{~mm})$

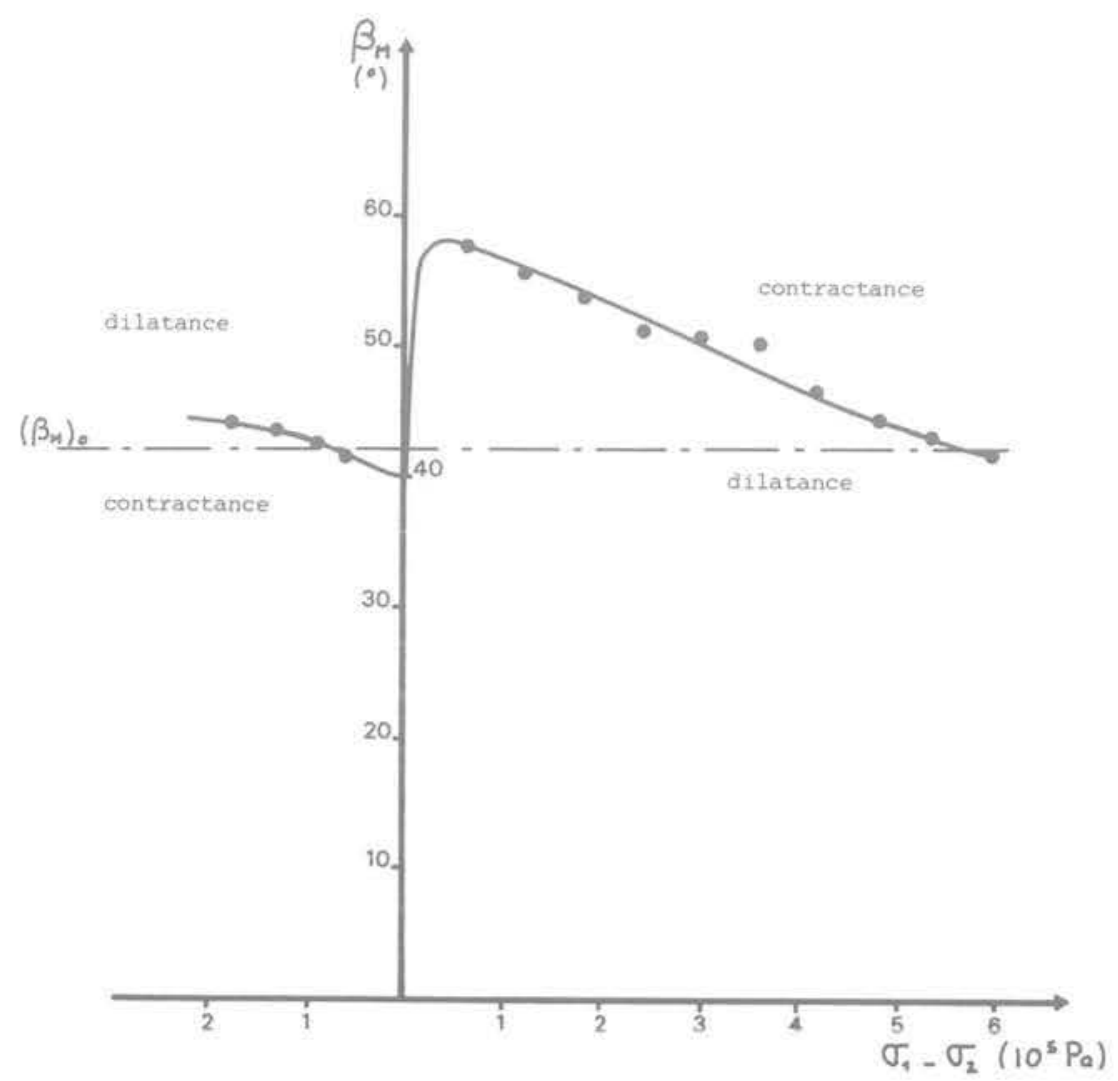

Fig. 23 Evolution de l'orientation moyenne des contacts glissants au cours d'une sollicitation biaxiale. Résultats obtenus par calcul numérique sur le modèle analogique. Matériau simplement déversé $(1-2 \mathrm{~mm})$ 


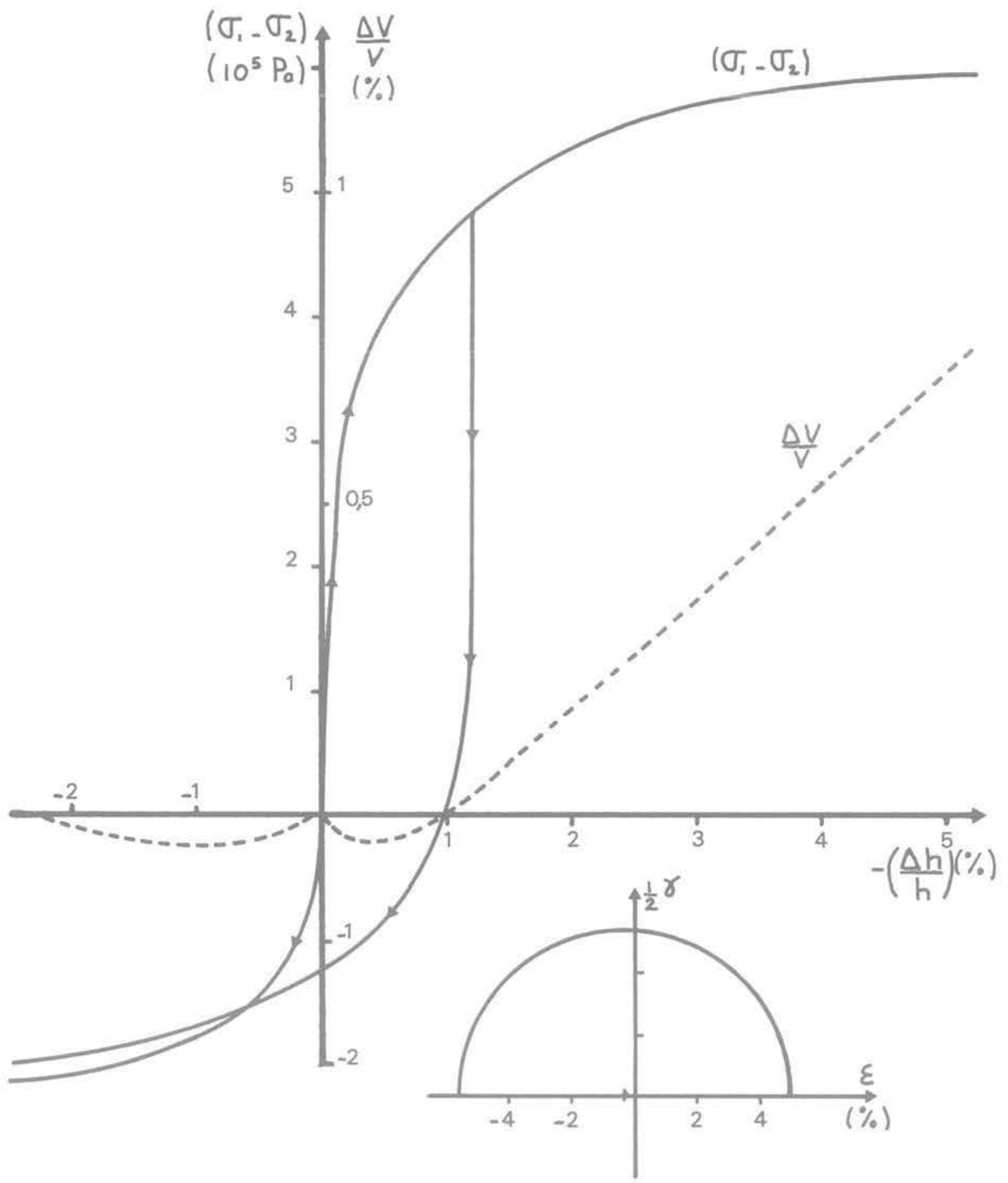

Fig. 24 Sollicitation biaxiale sur le matériau bidimensionnel $(1-2 \mathrm{~mm})$ simplement déversé. Résultats obtenus par calcul numérique sur le modèle analogique. 


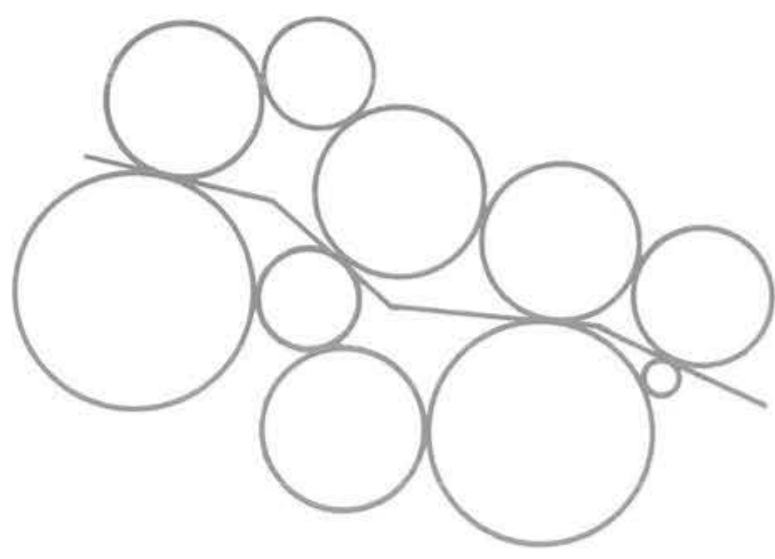

Bonnes conditions cinématiques pour les contacts glissants

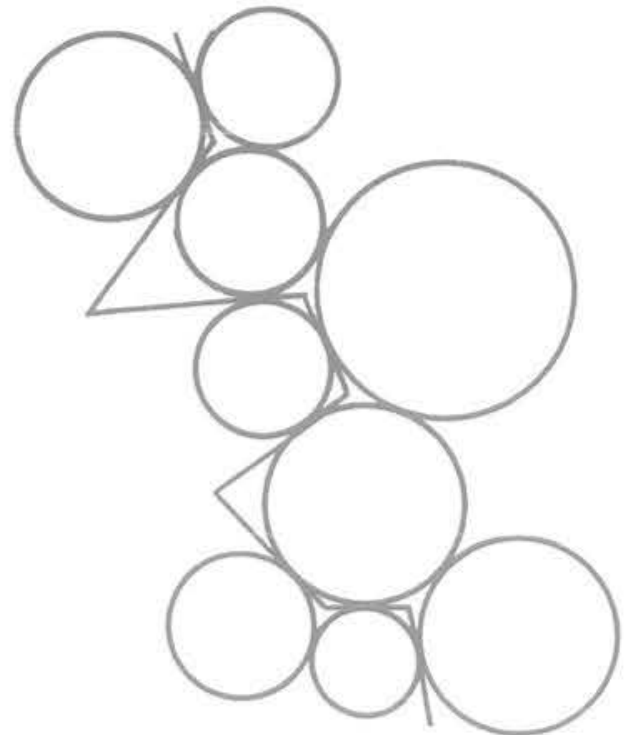

Mauvaises conditions cinématiques pour les contacts glissants

Fig. 25 Discontinuités cinématiques dans un milieu granulaire

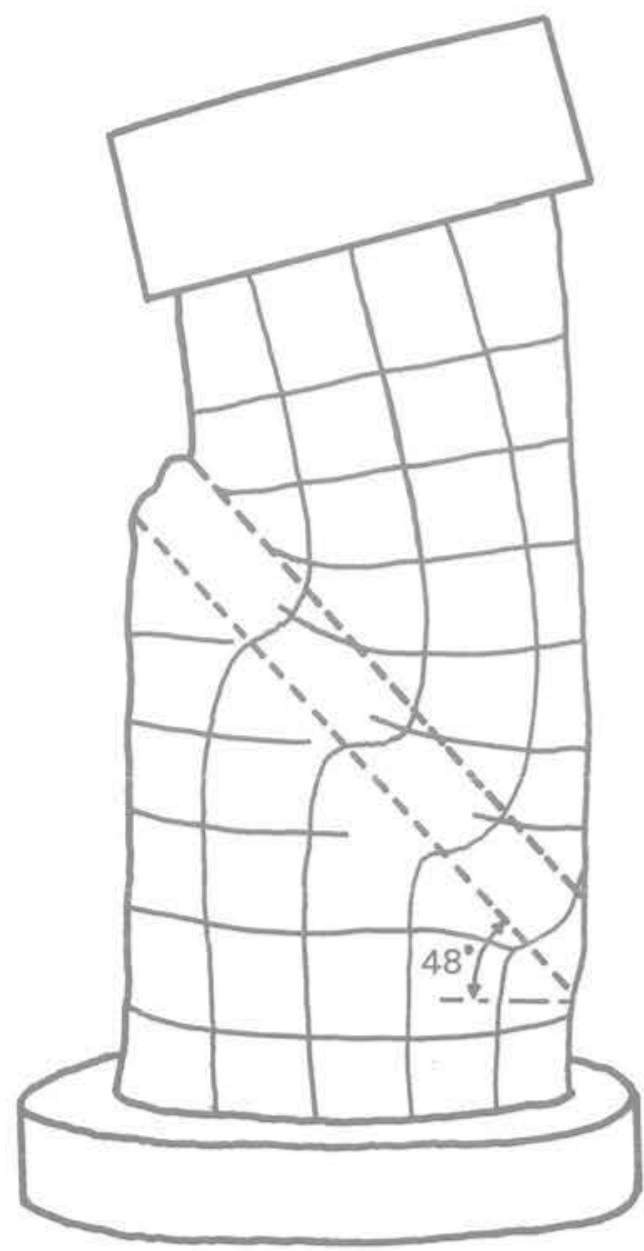

Fig. 26 Relevé d'après une photographie de la direction du plan de cisaillement au cours d'un essai triaxial sur du sable compact $\left(\Phi=35^{\circ}\right)$
- Loi d'écoulement plastique

Si on fait I'hypothèse que tout se passe comme si l'orientation de tous les contacts glissants était égale à leur valeur moyenne $\beta_{m}$ on obtient la loi de comportement plastique suivante, pour une sollicitation bidimensionnelle :

$$
\begin{aligned}
& \Delta \varepsilon_{z}=a_{1} m_{1 z} \sin \beta_{m} \\
& \Delta \varepsilon_{x}=-a_{1} m_{1 x} \cos \beta_{m}
\end{aligned}
$$

$a_{1}$ est un facteur arbitraire,

$\beta_{m}$ évolue au cours de la sollicitation.

- Discontinuités cinématiques

Du fait d'une hétérogénéité ou de conditions aux limites non uniformes on peut avoir localisation de grandes déformations suivant une ligne de discontinuité cinématique.

Soit $\beta_{r}$ l'orientation de cette ligne de discontinuité par rapport à $\mathrm{Ox}$, si elle existe, $\beta_{\mathrm{r}}$ correspond à I'orientation moyenne des directions de glissement aux points de contact.

Cette ligne de discontinuité, d'une part doit répondre à des conditions cinématiques sur les déplacements, et d'autre part doit se développer de façon à minimiser l'énergie nécessaire à sa création.

Conditions cinématiques optimales :

La ligne de glissement devra être la plus continue possible, sans brusque décrochement. Ce qui correspond à des valeurs de $\beta_{i}$ ayant toutes les mêmes signes (fig. 25), donc

$$
\beta_{r}=\beta_{m}=\left(\beta_{m}\right)_{0}+d
$$

$d>0$ pour un matériau contractant, $d<0$ pour un matériau dilatant. 
Conditions d'énergie minimale :

Le rapport de l'énergie dépensée par la composante verticale et la composante horizontale de la force de contact au point de contact $i$ est égal à :

$$
E_{i}=\frac{\operatorname{tg} \beta_{i}}{\operatorname{tg}\left(\beta_{i}-\Psi\right)}
$$

en première approximation on fait l'hypothèse que tout se passe comme si tous les contacts considérés présentaient l'orientation $\beta_{r}$

Le rapport de l'énergie dépensée par ${ }^{\circ}{ }_{1}$ et $\sigma_{3}$ pourra donc s'écrire :

$$
E=\frac{\operatorname{tg} \beta_{r}}{\operatorname{tg}\left[\beta_{r}-\Psi\right]}
$$

E est minimum pour

$$
\beta_{r}=\frac{\pi}{4}+\frac{\Psi}{2}
$$

on aura donc les deux conditions suivantes:

$$
\begin{aligned}
& \beta_{r}=\beta_{m}=\left(\beta_{m}\right)_{0}+d \\
& \beta_{r}=\frac{\pi}{4}+\frac{\Psi}{2} .
\end{aligned}
$$

Pour un milieu isotrope bidimensionnel, $\left(\beta_{m}\right)_{0}=45^{\circ}$, pour un milieu isotrope à 3 dimensions soumis à une sollicitation axisymétrique, $\left(\beta_{m}\right)_{0}=52^{\circ}$.

On aura donc, dans ce dernier cas :

$$
52^{\circ}+\mathrm{d}<\theta<45^{\circ}+\frac{\Psi}{2}
$$

Cette valeur de $\theta$ est donc toujours inférieure à $\frac{\pi}{4}+\frac{\phi}{2}$ (valeur habituellement admise). Effectivement les valeurs expérimentales mesurées sont toujours inférieures à $\left(\frac{\pi}{4}+\frac{\phi}{2}\right)$, par exemple sur la figure 26 , on peut observer un angle $\theta=48^{\circ}$ correspondant au cisaillement d'un matériau dilatant $(d<0)$. Dans ce cas il semble que $\theta$ soit proche de sa borne inférieure (correspondant aux conditions cinématiques optimales).

On notera aussi que pour un matériau dilatant $(d<0)$ les conditions cinématiques optimales correspondent à des valeurs de $\theta$ plus éloignées des conditions d'énergie minimum. La dilatance correspond à une dépense d'énergie, les matériaux dilatants présentent des angles de frottement plus élevés.

\section{Conclusions}

Le modèle présenté ici a permis d'analyser et parfois d'expliquer au niveau des contacts entre particules les phénomènes que l'on observe globalement au cours d'essais sur des matériaux granulaires, tels que l'anisotropie, l'irréversibilité, l'écrouissage, la dilatance, l'écoulement plastique, l'existence de discontinuités cinématiques.

Cette étude a pu aboutir à ces quelques résultats intéressants, d'une part, parce que nous avons simplifié le problème (particules cylindriques ou sphériques), d'autre part, grâce à un certain nombre d'hypothèses qui peuvent paraître parfois un peu arbitraires et ne trouvent de justification que globalement par la bonne concordance du modèle à la réalité.

Nous sommes conscients de ces limitations qui pourront pour certaines, être franchies au cours d'études ultérieures.

\section{Références bibliographiques}

Auvinet, G. (1975) : " Generation of granular media by computer». Proc. 5th Pan. Am. Conf. SMFE, Buenos Aires, vol. 1, p. 205.

Auvinet, G. (1977) : "Structure des milieux pulvérulents". IX Congr. int. de mécanique des sols et des fondations». Tokyo, vol. 1, p. 23-26.

Biarez, J. (1962): "Contribution à l'étude des propriétés mécaniques des sols et des matériaux pulvérulents". Thèse de doctorat ès Sciences, Grenoble.

Bishop, A.W. (1966): "The strengh of soils as engineering materials ". 6th Rankine lecture. Géotechnique $16 n^{\circ} 2$, p. $91-128$.

Cambou, B. (1977) : "Analyse statistique au niveau de la particule d'un milieu granulaire $n$. I $X^{e}$ Congr. int. de mécanique des sols et des fondations. Tokyo, session spéciale $n^{\circ} 5$.

Cambou, B. (1979) : «Approche du comportement d'un sol considéré comme un milieu non continu $n$. Thèse doctorat ès Sciences, Lyon.

Cundall, P.A., Strack, O.D.L. (1979): "A discrete numerical model for granular assemblies *. Geotechnique $29 n^{\circ} 1$, p. $47-65$.

Dantu, P. (1957) : “Contribution à l'étude mécanique et géométrique des milieux pulvérulents". IV Cong. int. de mécanique des sols et des fondations. Londres, p. 144-148.

Dantu, P. (1961): “Étude mécanique d'un milieu pulvérulent formé de sphères égales de compacité maximan, $V^{e}$ Cong. int. de mécanique des sois et des fondations, Paris.

De Josselin de Jong, G., Verruijt, A. (1969) : «Étude photo élastique d'un empilement de disques ". Cahiers du groupe français de Rhéologie $n^{\circ} 1$, tome II, p. 73-86.

Deresiewicz, H. (1958): «Mechanics of granular matter $*$. Advances in applied mechanics, vol. 5 , p. $233-306$.

Drescher, A. (1976) : “ An experimental investigation of flow rules for granular materials using optically sensitive glass particule». Geotechnique $26, n^{\circ} 4$, p. $591-601$.

Duffy, J., Mindlin, R.D. (1957) : « Stress-strain relations and vibrations of a granular medium". Journal of applied mechanics, p. 585-593.

Field, W.G. (1963) : « Towards a statistical definition of a granular mass". IVth A. and N.Z. conf. on soil mechanics.

Fletcher, E.H. (1971) : « Random walk of ideal granular mass ". Journ. soil mech. and F.D. Proc. ASCE SM 10, Oct., p. $1379-1392$.

Horne, M.R. (1969) : "The behaviour of an assembly of rotund, rigid, cohesionless particles». Proc. Roy. Soc. A, vol. 310, p. 24-34. 
Johnson, K.L. (1955) : "Surface interaction between elastically loaded bodies under tangential forces" Proc. Roy. Soc. A, vol. 230, p. 531-549.

Jowitt, P.W., Munro, J. (1975) : " The influence of void distribution and entropy on the engineering properties of granular mediaw. Proc. 2th int. conf. on appl. of statistics and probability in soil and structural eng., p. 365-386.

Litwiniszyn, J. (1963) : * The model of a random walk of particles adapted to researches on problems of mechanics of loose media *. Bul. aca. Sc. Pol. vol. XI $n^{\circ} 10$, p. $61-70$.

Marsal, R.J. (1965) : « Stochastic processes in the grain skeleton of soils $"$. Vi $\left.\right|^{e}$ cong. int. de mécanique des sols et des fondations. Montréal vol. 2, p. 303 à 307.

Marsal, R.J. (1973): "Mechanical properties of rockfill ». Embankment dam engineering. Casagrande volume (J. Wiley), p. 109 à 200.

Matsuoka, H. (1974) : «A microscopic study on shear mechanism of granular materials *. Soil and foundations, vol. $14 \mathrm{n}^{\circ} 1, \mathrm{p}, 29-43$.

Mogami, T. (1969) : « Mechanics of granular material as a particulated mass ". VII ${ }^{\circ}$ Cong. int. de mécanique des sols et des fondations. Mexico, p. 281-285.

Muramaya, S., Matsuoka, H. (1973) : « Mécanisme de cisaillement des sols $*$. VIII ${ }^{\circ}$ Cong. int. de mécanique des sols et des fondations. Moscou.

Newland, P.L., Allely, B.H. (1957) : "Volume changes in drained triaxial tests on granular materials ". Geotechnique $7 n^{\circ} 3$, p. 17-34.

Oda, M. (1974) : " A mechanical and statistical model of granular material $n$. Soils and foundations, vol. $14 n^{\circ} 1$, p. 13-27.
Oda, M., Konischi J. (1974) : « Microscopic deformation mechanism of granular material in simple shear $"$. Soils and foundations, vol. $14 n^{\circ} 4$, p. 25-38.

Rodriguez Ortiz, J. M. (1974) : « Estudio del comportamiento de medios granulares heterogeneos mediante modelos discontinuos analogicos y matematicos n. Thèse Université polytechnique de Madrid.

Rowe, P.W. (1962) : "The stress dilatancy relation for static equilibrium of an assembly of particles in contact w, Proc. Roy. Soc. A, vol. 269, p. 500-527.

Schlosser, F. (1972): "Comportement des milieux granulaires *. Bul. liaison P. et C. numéro spécial : "Le comportement des sols avant rupture», p. 37-47.

Schlosser, F. (1974) : «Influence des déformations des grains dans les milieux granulaires ». Bul. Liaison P. et C. $n^{\circ} 69, p \cdot 97-103$.

Smith. Foot, Busang (1929) : "Packing of homogeneous spheres». Physical review. Vol. 34, p. $1271-$ 1274.

Terzaghi, K. (1920) : "Old Earth Pressure theories and new test results". Eng. News Record. Vol. $85 n^{\circ} 14$, sept., p. 632-637.

Weber, J. (1966) ; « Recherche concernant les contraintes intergranulaires dans les milieux pulvérulents . Bul. liaison P. et C. $n^{\circ} 20$, p. $3.1-3.20$.

Wiendieck, K. (1964): "L'anisotropie des milieux pulvérulents et son influence sur les tassements". Thèse docteur-ingénieur. Grenoble.

Wilkins, J.K. (1970) : «A theory for the shear strenght of rockfill ». Rock mechanics, vol. 2, p. 205-222.

Wilson, R.J. (1962) : «Deformation of granular materials". Thèse Université de Newcastle Australie. 